\title{
Chemoenzymatic Synthesis of $\alpha$-Substituted Serines via Enantiodivergent Transformation
}

\author{
Shigeki Sano*, Michiyasu Nakao, Masanori Takeyasu, Chiyo Yamamoto, Syuji Kitaike, Yasuko \\ Yoshioka and Yoshimitsu Nagao
}

Graduate School of Pharmaceutical Sciences, The University of Tokushima, Sho-machi, Tokushima 770-8505, Japan

\begin{abstract}
A series of $(R)-N$-Cbz-2-alkyl-2-amino-3-hydroxypropyl acetates was prepared by enzymatic acetylation of $N$ Cbz-2-alkyl-2-aminopropane-1,3-diols with immobilized lipoprotein lipase from Pseudomonas sp. in up to $98 \%$ enantiomeric excess (ee). Enantiodivergent oxidation of $(R)$ - $N$-Cbz-2-alkyl-2-amino-3-hydroxypropyl acetates readily furnished $(R)$ - and $(S)$ - $\alpha$-substituted serines ( $\alpha$-benzylserines and $\alpha$-methylserines). Enzymatic hydrolysis of diethyl $N$-Cbz-2amino-2-methylmalonate catalyzed by porcine liver esterase afforded $(R)$ - $N$-Cbz-2-amino-3-ethoxy-2-methyl-3oxopropanoic acid in $97 \%$ ee. $(R)-N$-Cbz-2-amino-3-ethoxy-2-methyl-3-oxopropanoic acid was also transformed to both enantiomers of $\alpha$-methylserine via enantiodivergent reduction.
\end{abstract}

Keywords: Asymmetric synthesis, Lipoprotein lipase, Acetylation, 1,3-Diols, Enantiodivergent, $\alpha$-Substituted serines.

\section{INTRODUCTION}

In recent years there has been a considerable interest in the asymmetric synthesis of $\alpha, \alpha$-disubstituted $\alpha$-amino acids because of their biological importance [1]. The $\alpha$-substituted serine moiety has been of particular interest as a unique structure found in bioactive natural products, such as myriocin (ISP-I, thermozymocidin) [2], sphingofungin E [3], (+)conagenin [4], and (+)-lactacystin [5] (Fig. (1)). Thus, the asymmetric synthesis of $\alpha$-substituted serines has been an area of intensive investigation in our laboratory for the past few years.<smiles>[M]CCCCCC(=O)CCCCCC/C=C/[C@@H]([R])[C@@H](O)[C@H](O)[C@](N)(CO)C(=O)O</smiles><smiles>C[C@H](O)[C@@H](C)[C@H](O)C(=O)N[C@@](C)(CO)C(=O)O</smiles>

(+)-conagenin

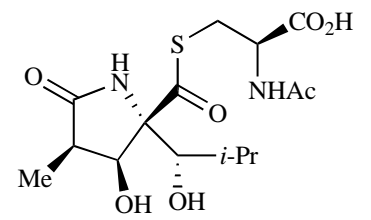

(+)-lactacystin
Fig. (1). Bioactive natural products including an $\alpha$-substituted serine moiety.

As part of our own contribution to this area, we developed several non-enzymatic asymmetric syntheses based on novel bislactim ethers derived from diethyl aminomalonate hydrochloride [6]. Further, we applied a chemo-enzymatic

*Address correspondence to this author at the Graduate School of Pharmaceutical Sciences, The University of Tokushima, Sho-machi, Tokushima 770-8505, Japan; Tel: +81-88-633-7273; Fax: +81-88-633-9503;

E-mail: ssano@ph.tokushima-u.ac.jp synthesis of $\alpha$-substituted serines utilizing enzyme-catalyzed desymmetrization of $\sigma$-symmetric compounds [7]. The synthesis of $\alpha$-substituted serines was accomplished by a combination of esterase-catalyzed enantioselective hydrolysis of prochiral diethyl $\mathrm{N}$-Cbz-2-alkyl-2-aminomalonates and enantiodivergent reduction of resultant acid esters. The enantiodivergent strategy is efficient for synthesizing the desired chiral $\alpha$-substituted serine without dependence on the absolute configuration of a single starting enantiomer [8]. However, the limited specificity of substrates is a disadvantage of this chemo-enzymatic reaction. The esterase-catalyzed hydrolysis of diethyl $\mathrm{N}$-Cbz-2-hexyl-2-aminomalonate gave only a trace amount of the corresponding acid ester. In addition, the moderate yields in the enantiodivergent transformation should be improved in this chemo-enzymatic synthesis. We therefore developed lipase-catalyzed enantioselective acetylation of prochiral $\mathrm{N}$-Cbz-2-alkyl-2-aminopropane-1,3diols [9]. The lipase-catalyzed acetylation of $\mathrm{N}$-Cbz-2amino-2-hexylpropane-1,3-diol provided satisfactory results.

In this paper, we describe in detail the lipase-catalyzed enantioselective acetylation of prochiral $\sigma$-symmetric $N$ Cbz-2-alkyl-2-aminopropane-1,3-diols and the enantiodivergent oxidation of the resultant chiral mono-acetylated product toward the construction of both enantiomers of $\alpha$ substituted serines as shown in Scheme 1. We also illustrate improved enantiodivergent reduction of $(R)-\mathrm{N}$-Cbz-2-amino2 -methylmalonate to both enantiomers of $\alpha$-methylserine (Scheme 1).

\section{RESULTS AND DISCUSSION}

$\mathrm{N}$-Cbz-2-alkyl-2-aminopropane-1,3-diols 4a-d, substrates for lipase-catalyzed acetylation, were synthesized as shown in Scheme 2. Diethyl $N$-Cbz-2-aminomalonate 2 was prepared from diethyl 2-aminomalonate hydrochloride 1 by a standard method in 97\% yield [6]. Then diethyl $\mathrm{N}$-Cbz-2alkyl-2-aminomalonates 3a-d were obtained by the treatment of $N$-protected diester 2 with $\mathrm{NaH}$, followed by alkylation of 

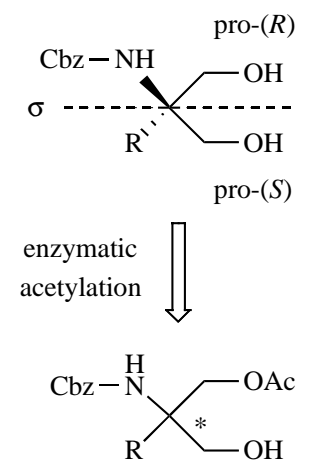

$(R)$ or $(S)$
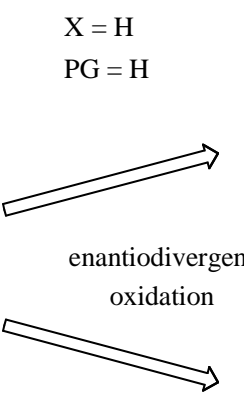

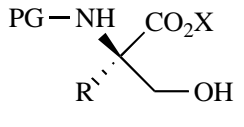

$\mathrm{X}=\mathrm{Et}$

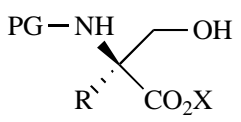

$(S)$-enantiomer

(R)-enantiomer

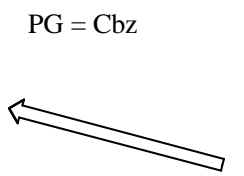

enantiodivergent

reduction

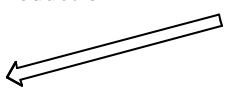

$\mathrm{R}=\mathrm{Bn}, \mathrm{Me}$, allyl

$\mathrm{R} \neq n$-hexyl

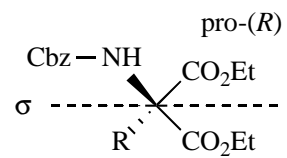

pro- $(S)$

enzymatic

hydrolysis

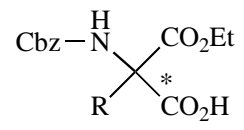

$(R)$ or $(S)$

$$
\mathrm{R}=\mathrm{Bn}, \mathrm{Me} \text {, allyl, } n \text {-hexyl }
$$

Scheme 1. Chemoenzymatic enantiodivergent synthesis of chiral $\alpha$-substituted serines.

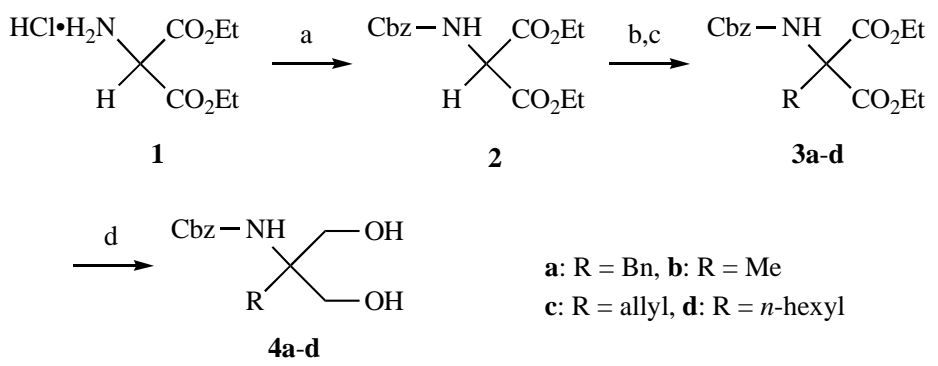

Scheme 2. Reagents and conditions: (a) Cbz-Cl, $\mathrm{NaHCO}_{3}, \mathrm{H}_{2} \mathrm{O}-\mathrm{Et}_{2} \mathrm{O}(1: 1), \mathrm{rt}, 6 \mathrm{~h}, 97 \%$; (b) $\mathrm{NaH}, \mathrm{THF}, 0{ }^{\circ} \mathrm{C}, 20 \mathrm{~min} ;(\mathrm{c})$ a: $\mathrm{BnBr}, \mathrm{THF}, 0$ ${ }^{\circ} \mathrm{C}, 20 \mathrm{~min}$ to rt, $5 \mathrm{~h}, 82 \%$, b: MeI, THF, rt, $15 \mathrm{~min}$ to $50{ }^{\circ} \mathrm{C}, 2 \mathrm{~h}, 87 \%$, c: $\mathrm{CH}_{2}=\mathrm{CHCH}_{2} \mathrm{Br}, \mathrm{THF}, 0{ }^{\circ} \mathrm{C}, 40 \mathrm{~min}$ to rt, $7 \mathrm{~h}, 88 \%$, d: $\mathrm{Me}\left(\mathrm{CH}_{2}\right)_{5} \mathrm{I}$, THF, $50{ }^{\circ} \mathrm{C}, 20 \mathrm{~h}, 77 \%$; (d) a: $\mathrm{LiBH}_{4}$, THF, $0{ }^{\circ} \mathrm{C}, 1 \mathrm{~h}$ to rt, 20 h, 59\%, b: $\mathrm{LiBH}_{4}, \mathrm{Et}_{2} \mathrm{O}, \mathrm{rt}, 24 \mathrm{~h}, 74 \%, \mathbf{c}: \mathrm{NaBH}_{4}, \mathrm{EtOH}, 0{ }^{\circ} \mathrm{C}, 19.5 \mathrm{~h}$ to rt, 8 h, $58 \%$, d: $\mathrm{LiBH}_{4}, \mathrm{THF}, 0{ }^{\circ} \mathrm{C}, 10 \mathrm{~h}$ to rt, $5 \mathrm{~h}, 48 \%$.

the resulting sodium enolate with alkyl halides in $77-88 \%$ yields. Subsequent reduction of diesters 3a-d with $\mathrm{LiBH}_{4}$ or $\mathrm{NaBH}_{4}$ afforded the corresponding 1,3-diols 4a-d in 48-74\% yields, respectively.

Lipase-catalyzed acetylation of the 1,3-diols 4a-d into the chiral monoacetates 5a-d was investigated utilizing more than 10 kinds of commercially available lipases (Scheme 3).

First, we attempted the acetylation of prochiral 1,3-diol 4b with vinyl acetate in the presence of several kinds of lipases in THF at room temperature. This yielded monoacetate 5b in 30-35\% enantiomeric excess (ee) with a series of Lipase PS from Pseudomonas sp. (Table 1, entries 4-6). The yield was significantly improved when an immobilized enzyme, such as Lipase PS-C or Lipase PS-D, was used. In addition, different kinds of lipases from Pseudomonas sp. improved both the yield and the ee (Table 1, entries 10-12).

Lipase-catalyzed acetylation of $\mathbf{4 b}$ was further investigated with LPL (lipoprotein lipase from Pseudomonas sp.)

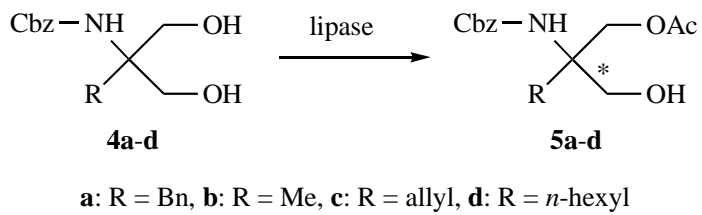

Scheme 3. Lipase-catalyzed acetylation of $\mathrm{N}$-Cbz-2-alkyl-2aminopropane-1,3-diols 4a-d (Tables 1-6). purchased from Fluka (Table $\mathbf{1}$, entry 12). The yields of $\mathbf{5} \mathbf{b}$ varied depending on the reaction temperature, which did not have as great an effect on the ee values (Table 2). The yield of monoacetate $\mathbf{5 b}$ was low at $-30{ }^{\circ} \mathrm{C}$, and the formation of diester by overacetylation was remarkable at $40{ }^{\circ} \mathrm{C}$. The overacetylation did not contribute to the desymmetrization of 1,3-diol 4b (Table 2, entries 4 and 5).

On the other hand, both the yield and the ee were influenced by the solvent used in the LPL-catalyzed acetylation in Table 3. In other words, acetylation in an acyclic ether (entries 3-7) was superior to that in a cyclic ether (entries 1 and 2). In particular, tert-butyl methyl ether (TBME) and cyclopentyl methyl ether (CPME) gave good results in both the yield and the ee (Table 3, entries 6 and 7). TBME is one of the ordinary solvents employed in enzyme-catalyzed acylation [10]. These reactions in Table 3 were quenched when the appearance of the corresponding diacetate was detected by TLC (silica gel).

The prochiral 1,3-diol $\mathbf{4 b}$ was also converted into the monoacetate $\mathbf{5 b}$ by LPL-catalyzed acylation with various acyl donors. Equally good results were obtained by utilizing isopropenyl acetate as an acyl donor instead of vinyl acetate (Table 4, entries 1 and 2).

Finally, optimized results of lipase-catalyzed acetylation of 1,3-diol 4a-d with vinyl acetate (4 mol eq) in TBME were achieved by employing LPL from Pseudomonas sp. (Fluka, lyophilized powder, ca. 20,000 units/mmol) or immobilized 
Table 1. Lipase-Catalyzed Acetylation of Propane-1,3-diol $4 b^{\text {a }}$

\begin{tabular}{|c|c|c|c|c|c|}
\hline Entry & $4 b$ & Lipase $^{\mathrm{b}}$ & Time & Yield of $5 b(\%)^{c}$ & Ee of $5 b(\%)^{d}$ \\
\hline 2 & $100 \mathrm{mg}$ & Lipase AYS & $48 \mathrm{~h}$ & 1 & $-^{\mathrm{e}}$ \\
\hline 3 & $100 \mathrm{mg}$ & Lipase AS & $48 \mathrm{~h}$ & 2 & 1 \\
\hline 4 & $100 \mathrm{mg}$ & Lipase PS & $24 \mathrm{~h}$ & 3 & $35(R)$ \\
\hline 5 & $100 \mathrm{mg}$ & Lipase PS-C & $24 \mathrm{~h}$ & 55 & $35(R)$ \\
\hline 7 & $100 \mathrm{mg}$ & Lipase from Pseudomonas cepacia & $5 \mathrm{~d}$ & 3 & $34(R)$ \\
\hline 8 & $100 \mathrm{mg}$ & Lipase from Pseudomonas fluorescens & $15 \mathrm{~h}$ & 38 & $28(R)$ \\
\hline 9 & $100 \mathrm{mg}$ & Lipoprotein lipase from Pseudomonas sp. & $14 \mathrm{~d}$ & 10 & $36(R)$ \\
\hline 10 & $80 \mathrm{mg}$ & Lipoprotein lipase from Pseudomonas sp. & $3 \mathrm{~h}$ & 78 & $72(R)$ \\
\hline
\end{tabular}

${ }^{a}$ Lipase (entries 1-6, 12,000 units/mmol; entries 7-12, $30 \mathrm{mg}$ ), vinyl acetate (5 mol eq), THF (10 ml), rt.

${ }^{\mathrm{b}}$ Entries 1-6: Amano Enzyme. PS-C: immobilized on ceramic particles. PS-D: immobilized on diatomaceous earth. Entries 7, 8, 12: Fluka; entry 9: Wako Pure Chemical Industries; entry 10: Toyobo; entry 11: Sigma.

${ }^{c}$ Isolated yields.

${ }^{\mathrm{d}}$ HPLC analysis (CHIRALPAK AD-H, $n$-hexane/2-propanol = 15/1, $1.0 \mathrm{ml} / \mathrm{min}, 254 \mathrm{~nm}$ ).

${ }^{\mathrm{e}}$ Not determined.

Table 2. LPL-Catalyzed Acetylation of Propane-1,3-diol $4 b^{a}$

\begin{tabular}{|c|c|c|c|c|c|}
\hline Entry & Temperature & Time & Yield of $5 b(\%)^{b}$ & Recovery of $4 b(\%)^{b}$ & Ee of $5 b(\%)^{c}$ \\
\hline 2 & $0{ }^{\circ} \mathrm{C}$ & $7 \mathrm{~h}$ & 58 & 36 & $76(R)$ \\
\hline 3 & $-30^{\circ} \mathrm{C}$ & $2 \mathrm{~d}$ & 22 & 78 & $76(R)$ \\
\hline 4 & $40{ }^{\circ} \mathrm{C}$ & $30 \mathrm{~min}$ & 66 & $21^{\mathrm{d}}$ & $72(R)$ \\
\hline
\end{tabular}

${ }^{\mathrm{a}} \mathbf{4 b}(100 \mathrm{mg}), \mathrm{LPL}(30 \mathrm{mg})$, vinyl acetate $(5 \mathrm{~mol} \mathrm{eq})$, THF $(10 \mathrm{ml})$

bIsolated yields.

${ }^{\mathrm{c} H P L C}$ analysis (CHIRALPAK AD-H, $n$-hexane/2-propanol = 15/1 or 30/1, $1.0 \mathrm{ml} / \mathrm{min}, 254 \mathrm{~nm}$ ).

${ }^{\mathrm{d}}$ Diacetate $(11 \%)$ was obtained.

${ }^{\mathrm{e}}$ Diacetate $(50 \%)$ was obtained.

Table 3. LPL-Catalyzed Acetylation of Propane-1,3-diol $4 b^{a}$

\begin{tabular}{|c|c|c|c|c|c|}
\hline Entry & Solvent ${ }^{b}$ & Time (h) & Yield of $5 b(\%)^{c}$ & Recovery of $4 b(\%)^{c}$ & Ee of $5 b(\%)^{d}$ \\
\hline 1 & THF & 1.5 & 85 & 9 & $78(R)$ \\
\hline 2 & 1,4-dioxane & 2 & 69 & 19 & $72(R)$ \\
\hline 3 & $\mathrm{Et}_{2} \mathrm{O}$ & 4 & 56 & 43 & $85(R)$ \\
\hline 4 & DBE & 5 & 17 & 37 & $88(R)$ \\
\hline 5 & DIPE & 4 & 23 & 55 & $89(R)$ \\
\hline 6 & TBME & 6 & 75 & 10 & $85(R)$ \\
\hline 7 & CPME & 6 & 79 & 11 & $86(R)$ \\
\hline 8 & benzene & 12 & 25 & 61 & $82(R)$ \\
\hline 9 & dichloromethane & 6 & 12 & 84 & $78(R)$ \\
\hline 10 & $-^{\mathrm{e}}$ & 1.5 & 26 & 74 & $85(R)$ \\
\hline
\end{tabular}

${ }^{\mathrm{a}} 4 \mathbf{b}(100 \mathrm{mg})$, LPL (30 mg), vinyl acetate $(5 \mathrm{~mol} \mathrm{eq})$, solvent $(10 \mathrm{ml})$, rt.

${ }^{\mathrm{b}}$ DBE: di- $n$-butyl ether; DIPE: diisopropyl ether; TBME: tert-butyl methyl ether; CPME: cyclopentyl methyl ether.

${ }^{c}$ Isolated yields.

${ }^{\mathrm{d}}$ HPLC analysis (CHIRALCEL OD-H, $n$-hexane/2-propanol = 10/1, $1.0 \mathrm{ml} / \mathrm{min}, 254 \mathrm{~nm}$ ).

${ }^{\mathrm{e}}$ Vinyl acetate $(10 \mathrm{ml})$ was used as solvent. 
Table 4. LPL-Catalyzed Acylation of Propane-1,3-diol $4 b^{a}$

\begin{tabular}{|c|c|c|c|c|c|}
\hline Entry & Acyl Donor & Time (h) & Yield $(\%)^{b}$ & Recovery of $4 b(\%)^{b}$ & $\operatorname{Ee}(\%)^{\mathrm{c}}$ \\
\hline 1 & vinyl acetate & 6 & $75(5 \mathbf{b})$ & 10 & $85(\mathbf{5 b})(R)$ \\
\hline 3 & vinyl 2-chloroacetate & 8 & $53(\mathbf{5 b a})$ & 34 & $44(\mathbf{5 b a})$ \\
\hline 4 & vinyl butyrate & 3 & $58(\mathbf{5 b b})$ & 0 & $83(\mathbf{5 b b})$ \\
\hline 6 & vinyl pivalate & 6 & $12(\mathbf{5 b d})$ & 86 & $59(\mathbf{5 b d})$ \\
\hline
\end{tabular}

${ }^{\mathrm{a}} 4 \mathrm{~b}(100 \mathrm{mg})$, LPL (30 mg), acyl donor (5 mol eq), TBME (10 ml), rt.

${ }^{\mathrm{b}}$ Isolated yields.

${ }^{c}$ HPLC analysis (CHIRALCEL OD-H, $n$-hexane/2-propanol =10/1 or 19/1, $1.0 \mathrm{ml} / \mathrm{min}, 254 \mathrm{~nm}$ ).

Table 5. LPL-Catalyzed Acetylation of Propane-1,3-diol $4^{\mathrm{a}}$

\begin{tabular}{|c|c|c|c|c|c|c|c|}
\hline Entry & 4 & Lipase & Time (h) & Yield of $5(\%)^{b}$ & $\begin{array}{l}\text { Yield of Diace- } \\
\text { tate }(\%)^{\mathbf{b , c}}\end{array}$ & $\begin{array}{c}\text { Recovery of } 4 \\
(\%)^{b}\end{array}$ & Ee of $5(\%)$ \\
\hline 1 & $844 \mathrm{mg}(\mathbf{4 a})$ & LPL & 18 & $92(\mathbf{5 a})$ & 3 & $4(\mathbf{4 a})$ & $95(\mathbf{5 a})(R)^{\mathrm{d}}$ \\
\hline 2 & 137 mg (4b) & LPL & 3 & $73(\mathbf{5 b})$ & 23 & $1(\mathbf{4 b})$ & $88(\mathbf{5 b})(R)^{\mathrm{e}}$ \\
\hline 3 & $152 \mathrm{mg}(\mathbf{4 c})$ & LPL & 5.5 & $83(5 c)$ & 7 & $5(4 \mathrm{c})$ & $96(\mathbf{5 c})^{\mathrm{f}}$ \\
\hline 5 & 180 mg (4a) & Immobilized LPL & 2 & $94(\mathbf{5 a})$ & 3 & $1(\mathbf{4 a})$ & $97(\mathbf{5 a})(R)^{\mathrm{d}}$ \\
\hline 6 & $2.65 \mathrm{~g}(\mathbf{4 b})$ & Immobilized LPL & 1.3 & $72(\mathbf{5 b})$ & 26 & $2(4 \mathbf{b})$ & $92(\mathbf{5 b})(R)^{\mathrm{e}}$ \\
\hline 7 & 717 mg (4c) & Immobilized LPL & 1 & $90(\mathbf{5 c})$ & 3 & $5(4 c)$ & $98(\mathbf{5 c})^{\mathrm{f}}$ \\
\hline 8 & 177 mg (4d) & Immobilized LPL & 2 & $82(5 d)$ & 12 & $2(\mathbf{4 d})$ & $96(\mathbf{5 d})^{g}$ \\
\hline
\end{tabular}

${ }^{a}$ LPL (entry 1: 20,549 units/mmol; entries 2-4: 21,291 units/mmol; entries 5-8: 51 units $/ \mathrm{mmol}$ ), vinyl acetate (4 mol eq), TBME, rt.

${ }^{\mathrm{b}}$ Isolated yields.

${ }^{\mathrm{C}} \mathrm{N}$-Cbz-2-alkyl-2-aminopropane-1,3-diyl diacetate.

${ }^{\mathrm{d} H P L C}$ analysis (CHIRALCEL OJ-H, $n$-hexane $/ 2$-propanol $=5 / 1,1.0 \mathrm{ml} / \mathrm{min}, 254 \mathrm{~nm}$ ).

${ }^{\mathrm{e}} \mathrm{HPLC}$ analysis (CHIRALCEL OD-H, $n$-hexane $/ 2$-propanol $=10 / 1,1.0 \mathrm{ml} / \mathrm{min}, 254 \mathrm{~nm}$ ).

${ }^{\mathrm{f}}$ HPLC analysis (CHIRALPAK AD-H, $n$-hexane $/ 2$-propanol $=19 / 1,1.0 \mathrm{ml} / \mathrm{min}, 254 \mathrm{~nm}$ ).

${ }^{g} \mathrm{HPLC}$ analysis (CHIRALCEL OD-H, $n$-hexane $/ 2$-propanol = 19/1,1.0 ml/min, $254 \mathrm{~nm}$ ).

Table 6. Recycling of the Immobilized LPL in the LPL-Catalyzed Acetylation of Propane-1,3-diol 4c ${ }^{\mathrm{a}}$

\begin{tabular}{|c|c|c|c|c|c|c|c|}
\hline $\begin{array}{c}\text { Cycle } \\
\text { No. }\end{array}$ & 4c & Time (h) & Yield of 5c (\%) & $\begin{array}{c}\text { Yield of Diacetate } \\
(\boldsymbol{\%})^{\mathbf{b}, \mathbf{c}}\end{array}$ & $\begin{array}{c}\text { Recovery of } \\
\mathbf{4 c}(\boldsymbol{\%})^{\mathbf{b}}\end{array}$ & $\begin{array}{c}\text { Ee of 5c(\%) } \\
\text { d }\end{array}$ & $\begin{array}{c}\text { Recovery of Immobilized } \\
\text { LPL (\%) }\end{array}$ \\
\hline \hline 1 & $717 \mathrm{mg}$ & 1 & 90 & 3 & 5 & 98 & 87 \\
\hline 2 & $514 \mathrm{mg}$ & 5 & 84 & 6 & 3 & 97 & 84 \\
\hline 3 & $295 \mathrm{mg}$ & 22 & 84 & 4 & 3 & 97 & 72 \\
\hline
\end{tabular}

${ }^{\mathrm{a}}$ Immobilized LPL (51 units/mmol), vinyl acetate (4 mol eq), TBME, rt.

${ }^{\mathrm{b}}$ Isolated yields.

${ }^{\mathrm{c}} N$-Cbz-2-allyl-2-aminopropane-1,3-diyl diacetate.

${ }^{\mathrm{d}}$ HPLC analysis (CHIRALPAK AD-H, $n$-hexane $/ 2$-propanol = 19/1, $1.0 \mathrm{ml} / \mathrm{min}, 254 \mathrm{~nm}$ ).

LPL from Pseudomonas sp. [Toyobo, LPL from Pseudomonas sp. immobilized on Hyflo Super-Cel (diatomaceous earth), 51 units $/ \mathrm{mmol}$ ] at room temperature (Table 5).

The enantiomeric excess of monoacetates 5a-d was determined by means of HPLC analysis on a chiral-stationaryphase (CSP) in comparison with racemic monoacetates 5a-d. In addition, we attempted to recycle the immobilized LPL in the acetylation of propane-1,3-diol 4c. The results demon- strated that the LPL retained remarkable acetylation activity for at least three cycles (Table 6).

The absolute configuration of chiral monoacetates $5 \mathbf{a}$ and $\mathbf{5 b}$ was determined to be $R$ by their chemical conversion into the known chiral compounds $(R)$ - and $(S)-9 \mathbf{a}, \mathbf{b}$ and by comparing their specific rotations with those given in the literature (Scheme 4 and 5) [11,12]. Thus, the pro- $(R)$ hydroxymethyl group of $\mathbf{4 a}$ and $\mathbf{4 b}$ was selectively acetylated 


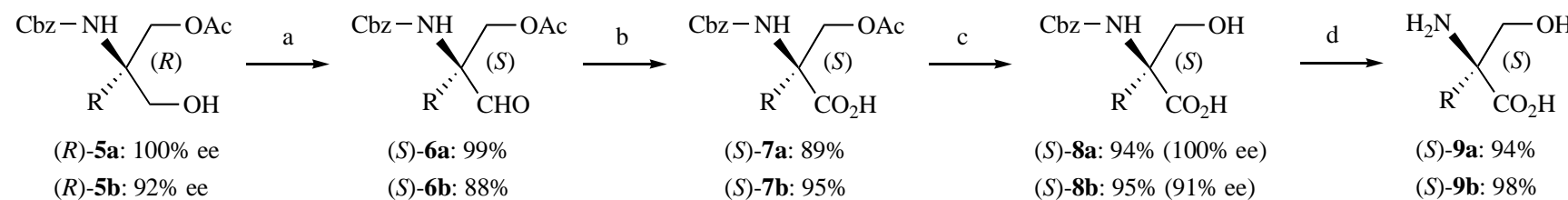

a: $\mathrm{R}=\mathrm{Bn}, \mathbf{b}: \mathrm{R}=\mathrm{Me}$

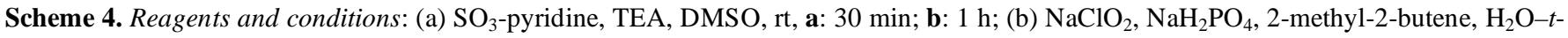
$\mathrm{BuOH}(1: 4)$, rt, a: 1 h; b: 1.5 h; (c) $\mathrm{LiEt}_{3} \mathrm{BH}$, THF, $0{ }^{\circ} \mathrm{C}$, a: $30 \mathrm{~min}$; b: $40 \mathrm{~min}$; (d) $\mathrm{H}_{2}, 10 \%$ Pd-C, EtOH, rt, 3.5 h.
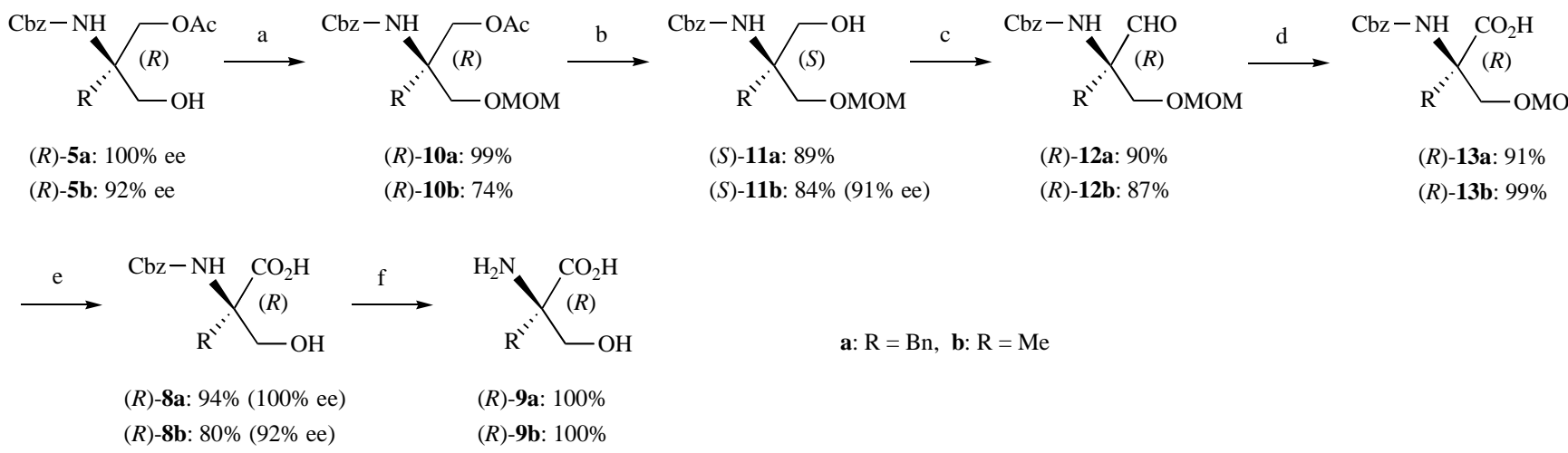

a: $\mathrm{R}=\mathrm{Bn}, \mathrm{b}: \mathrm{R}=\mathrm{Me}$

Scheme 5. Reagents and conditions: (a) MOMCl, DIPEA, a: $\mathrm{CH}_{2} \mathrm{Cl}_{2}, 0{ }^{\circ} \mathrm{C}, 30 \mathrm{~min}$ to rt, $20.5 \mathrm{~h}$; b: DMSO, rt, $7 \mathrm{~h}$; (b) a: $\mathrm{LiEt}{ }_{3} \mathrm{BH}, \mathrm{THF}, 0$ ${ }^{\circ} \mathrm{C}, 50 \mathrm{~min}$; b: $\mathrm{K}_{2} \mathrm{CO}_{3}, \mathrm{MeOH}$, rt, $10 \mathrm{~min}$; (c) $\mathrm{SO}_{3}$-pyridine, TEA, DMSO, rt, a: $40 \mathrm{~min}$; b: 50 min; (d) $\mathrm{NaClO}_{2}, \mathrm{NaH}_{2} \mathrm{PO}_{4}, 2-\mathrm{methyl}_{-2}-$ butene, $\mathrm{H}_{2} \mathrm{O}-t-\mathrm{BuOH}(1: 5)$, rt, a: 1 h; b: 1.5 h; (e) $1 \mathrm{~N} \mathrm{HCl}-\mathrm{THF}$ (1:2), reflux, a: 24 h; b: 21 h; (f) $\mathrm{H}_{2}, 10 \%$ Pd-C, EtOH, rt, a: 4 h; b: 3.5 h.

by LPL-catalyzed acetylation. The absolute configuration of the other chiral monoacetates, $\mathbf{5 c}$ and $\mathbf{5 d}$, was determined by utilizing the novel diketopiperazine method (the DKP method) proposed by us [13].

Enantiodivergent transformation of $(R)-5 \mathbf{a}$ to $(S)$ - $\alpha$ benzylserine $[(S)-9 \mathbf{a}]$ was performed without isomerization, as shown in Scheme 4. Recrystallization of $(R)-5 \mathbf{a}(97 \%$ ee) from AcOEt- $n$-hexane gave $(R)-5 \mathbf{a}$ in an enantiomerically pure form. Oxidation of $(R)$-5a with $\mathrm{SO}_{3}$-pyridine in the presence of triethylamine (TEA) gave aldehyde $(S)$-6a in $99 \%$ yield. Further oxidation of $(S)$-6a with $\mathrm{NaClO}_{2}$ afforded carboxylic acid (S)-7a in $89 \%$ yield [14]. Reductive deprotection of the acetyl group in (S)-7a was accomplished using $\mathrm{LiEt}_{3} \mathrm{BH}$ (Super Hydride ${ }^{\circledR}$ ) [15]. Namely, the corresponding alcohol $(S)$-8a was prepared in $94 \%$ yield and $100 \%$ ee. The enantiomeric excess of $(S)$-8a was determined by means of HPLC analysis on a CSP after quantitative methylation with an excess amount of (trimethylsilyl)diazomethane $\left(\mathrm{TMSCHN}_{2}\right)$ [16]. Catalytic hydrogenolysis of $(S)$-8a with $\mathrm{Pd}-\mathrm{C}$ under hydrogen at room temperature furnished the corresponding $\alpha$-benzylserine $(S)$-9a in $94 \%$ yield as an enantiomerically pure form.

On the other hand, enantiodivergent synthesis of $(R)-\alpha-$ benzylserine [ $(R)-\mathbf{9 a}]$ from $(R)-5 \mathbf{a}$ was achieved as shown in Scheme 5. Treatment of $(R)-5$ a with chloromethyl methyl ether $(\mathrm{MOMCl})$ in the presence of diisopropylethylamine (DIPEA) furnished $(R)-\mathbf{1 0}$, and then reductive deprotection of the acetyl group of the resulting $(R)$-10 provided the desired alcohol $(S)-\mathbf{1 1}$ in $88 \%$ yield (two steps). After oxidation of $(S)$-11 with $\mathrm{SO}_{3}$-pyridine in the presence of TEA, the resulting aldehyde $(R)-\mathbf{1 2}$ was subjected to further oxidation to give carboxylic acid $(R)-\mathbf{1 3}$ in $82 \%$ yield (two steps). Treatment of $(R)-\mathbf{1 3}$ in $1 \mathrm{~N} \mathrm{HCl}-\mathrm{THF}(1: 2)$ under reflux afforded the corresponding alcohol $(R)-\mathbf{8 a}$ in $94 \%$ yield and $100 \%$ ee. $\alpha$-Benzylserine $(R)$-9a was quantitatively prepared from $(R)$ 8a by catalytic hydrogenolysis.

$(S)$ - and $(R)$ - $\alpha$-methylserine [(S)- and $(R)-9 \mathbf{b}]$ was also obtained from $(R)-\mathbf{5 b}$ in a similar enantiodivergent transformation without isomerization, as shown in Schemes $\mathbf{4}$ and $\mathbf{5}$. In these reactions, deacetylation of $(R)-\mathbf{1 0 b}$ took place under mild conditions utilizing $\mathrm{K}_{2} \mathrm{CO}_{3}$.

Although LPL-catalyzed acetylation of 1,3-diol $\mathbf{4 b}$ afforded the corresponding monoacetate $(R)-\mathbf{5 b}$ in $92 \%$ ee as already shown in Table $\mathbf{5}$, we have previously reported that porcine liver esterase (PLE)-catalyzed hydrolysis of diester $\mathbf{3 b}$ and enantiodivergent reduction of the resultant acid ester $(R)$-14 furnished the corresponding $\alpha$-methylserine derivative $(R)$ - and $(S)-\mathbf{1 5}$ in $97 \%$ ee and $96 \%$ ee, respectively [7]. In this enzymatic hydrolysis of diester $\mathbf{3 b},(R)-\mathbf{1 4}$ was obtained in $97 \%$ ee (Scheme 6). Thus, we reinvestigated the enantiodivergent transformation of $(R)-\mathbf{1 4}$ to $(R)$ - and $(S)$-15. Chemoselective reduction of $(R)-\mathbf{1 4}$ with Super Hydride ${ }^{\circledR}$ in THF instead of $\mathrm{LiBH}_{4}$ in $\mathrm{Et}_{2} \mathrm{O}$ furnished $(S)-8 \mathbf{b}$ in $79 \%$ yield. Esterification of $(S)$-8b gave $(S)$-15 in $84 \%$ yield and $96 \%$ ee as shown in Scheme 7. On the other hand, fluorination of

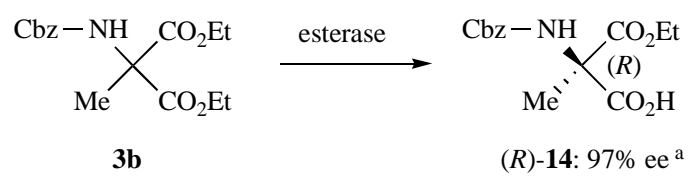

Scheme 6. Reagents and conditions: PLE (1,293 units/mmol), 1/15 M phosphate buffer ( $\mathrm{pH} 7.0)-\mathrm{MeCN}(9: 1), \mathrm{rt}, 6 \mathrm{~h}$. ${ }^{\mathrm{a}} \mathrm{HPLC}$ analysis (CHIRALCEL OD-H, $n$-hexane $/ 2$-propanol $=15 / 1,1.0 \mathrm{ml} / \mathrm{min}$, $254 \mathrm{~nm}$ ) after methylation with $\mathrm{TMSCHN}_{2}$. 


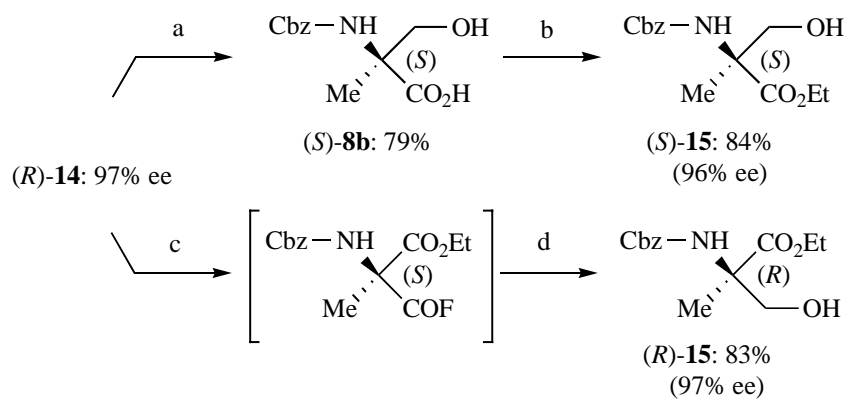

Scheme 7. Reagents and conditions: (a) $\mathrm{LiEt}_{3} \mathrm{BH}$, THF, $0{ }^{\circ} \mathrm{C}, 40 \mathrm{~min}$; (b) EtI, $\mathrm{K}_{2} \mathrm{CO}_{3}$, acetone, reflux, 2 h; (c) DAST, THF, rt, 2 h; (d) $\mathrm{NaBH}_{4}, \mathrm{CH}_{2} \mathrm{Cl}_{2}-\mathrm{MeOH}(2: 5),-78{ }^{\circ} \mathrm{C}, 1 \mathrm{~h}$.

$(R)-14$ with diethylaminosulfur trifluoride (DAST) in THF, followed by reduction of the resultant acyl fluoride with $\mathrm{NaBH}_{4}$ in $\mathrm{CH}_{2} \mathrm{Cl}_{2}-\mathrm{MeOH}$, afforded $(R)-\mathbf{1 5}$ in $83 \%$ yield and $97 \%$ ee [17]. The enantiomeric excess of $(R)$ - or $(S)-\mathbf{1 5}$ was determined by means of HPLC analysis on a CSP.

\section{CONCLUSIONS}

In conclusion, LPL-catalyzed acetylation of 1,3-diols 4ad gave the corresponding monoacetates 5a-d in $92-98 \%$ ee. Both enantiomers of $\alpha$-benzylserine [ $(R)$ - and $(S)-9 \mathbf{a}]$ and $\alpha$ methylserine $[(R)$ - and $(S)$-9b] were obtained by enantiodivergent oxidation of $(R)-\mathbf{5 a}$ and $(R)-\mathbf{5} \mathbf{b}$ without any isomerization. In addition, reinvestigation of the enantiodivergent transformation of $(R)-\mathbf{1 4}$ to $(R)$ - and $(S)$-15 resulted in optimization of our previously reported method. The development of applications exploiting the synthesis of novel functional molecules containing $\alpha$-substituted serines is currently underway in our laboratory.

\section{EXPERIMENTAL SECTION}

\section{General Information}

All melting points were determined on a Yanaco micro melting point apparatus and are uncorrected. IR spectra were obtained using a JASCO FT/IR-420 IR Fourier transform spectrometer. ${ }^{1} \mathrm{H}$ NMR (400 or $300 \mathrm{MHz}$ ) and ${ }^{13} \mathrm{C}$ NMR (100 or $75 \mathrm{MHz}$ ) spectra were recorded on JEOL JNMAL400 and JEOL JNM-AL300 spectrometers, respectively. Chemical shifts are given in $\delta$ values (ppm) using tetramethylsilane (TMS) as an internal standard. Fast atom bombardment mass spectra (FABMS) and electron impact mass spectra (EIMS) were recorded on a JEOL JMS SX102A spectrometer. Electron spray ionization mass spectra (ESIMS) were recorded on a Waters LCT Premier spectrometer. Elemental combustion analyses were performed using a Yanagimoto CHN CORDER MT-5. HPLC analyses were performed using a JASCO PU-980 apparatus equipped with a JASCO UV/VIS detector, using CHIRALPAK AD-H, CHIRALCEL OD-H or CHIRALCEL OJ-H (Daicel Chemical Industries). All reactions were monitored by TLC employing 0.25-mm silica gel plates (Merck 5715; $60 \mathrm{~F}_{254}$ ). Preparative TLC (PTLC) was performed on $0.5-\mathrm{mm}$ silica gel plates (Merck 5744; $60 \mathrm{~F}_{254}$ ). Column chromatography was carried out on silica gel [Silica Gel 60N (Kanto Chemical; spherical, neutral, 63-210 $\mu \mathrm{m}$ ), COSMOSIL 75 SL-IIPREP (Nacalai Tesque; spherical, 42-105 $\mu \mathrm{m}$ ) or Silica Gel 60 (Merck; 40-63 $\mu \mathrm{m}$ )]. Anhydrous THF, $\mathrm{CH}_{2} \mathrm{Cl}_{2}, \mathrm{MeOH}$, and DMF were used as purchased from Kanto Chemical. Anhydrous DMSO was commercially obtained from Wako
Pure Chemical Industry. All other reagents were used as purchased.

\section{Diethyl 2-(Benzyloxycarbonylamino)malonate (2)}

To a stirring solution of diethyl 2-aminomalonate hydrochloride (500 mg, $2.36 \mathrm{mmol})$ and $\mathrm{NaHCO}_{3}(515 \mathrm{mg}, 6.14$ $\mathrm{mmol})$ in $\mathrm{H}_{2} \mathrm{O}(15 \mathrm{~mL})$ was added $\mathrm{Cbz}-\mathrm{Cl}(219 \mu \mathrm{L}, 1.53$ $\mathrm{mmol})$ and $\mathrm{Et}_{2} \mathrm{O}(15 \mathrm{~mL})$. After being stirred at room temperature for $2 \mathrm{~h}, \mathrm{NaHCO}_{3}(515 \mathrm{mg}, 6.14 \mathrm{mmol})$ and $\mathrm{Cbz}-\mathrm{Cl}$ $(219 \mu \mathrm{L}, 1.53 \mathrm{mmol})$ were added, and the resulting mixture was stirred at room temperature for $4 \mathrm{~h}$. The solvent was removed in vacuo, AcOEt $(30 \mathrm{~mL})$ was added to the oily residue and the resultant solution was washed with $5 \% \mathrm{HCl}$ $(10 \mathrm{~mL}), \mathrm{H}_{2} \mathrm{O}(10 \mathrm{~mL})$, dried over anhydrous $\mathrm{MgSO}_{4}$, filtered, and concentrated in vacuo. The oily residue was purified by silica gel column chromatography [Silica Gel 60, $n$ hexane-AcOEt (2:1)] to afford $2(711 \mathrm{mg}, 97 \%)$ as a colorless oil. ${ }^{1} \mathrm{H}$ NMR $\left(400 \mathrm{MHz}, \mathrm{CDCl}_{3}\right) \delta 1.08-1.05(\mathrm{~m}, 6 \mathrm{H})$, 4.03-4.53 (m, 4H), 5.00 (d, J=7.5 Hz, 1H), 5.13 (s, 2H), 5.80 $(\mathrm{d}, J=6.8 \mathrm{~Hz}, 1 \mathrm{H}), 7.06-7.83(\mathrm{~m}, 5 \mathrm{H}) ;{ }^{13} \mathrm{C} \mathrm{NMR}(100 \mathrm{MHz}$, $\left.\mathrm{CDCl}_{3}\right) \delta 14.0,57.7,62.5,67.3,128.0,128.1,128.4,135.8$, 155.2, 166.1; IR (neat) 3376, 2985, 2361, 1505, 1217, 1026, $742 \mathrm{~cm}^{-1}$; EIMS calcd for $\mathrm{C}_{15} \mathrm{H}_{19} \mathrm{NO}_{6}$ MW 309.1212, found $m / z$ 309.1209 $\left(\mathrm{M}^{+}\right)$; Anal. Calcd for $\mathrm{C}_{15} \mathrm{H}_{19} \mathrm{NO}_{6}$ : C, 58.25; $\mathrm{H}$, $6.19 ; \mathrm{N}, 4.53$. Found: C, 58.21; H, 6.17; N, 4.40\%.

\section{Alkylation of Diethyl 2-(Benzyloxycarbonylamino) malonate (2) with Benzyl Bromide}

A solution of $2(3.0 \mathrm{~g}, 9.7 \mathrm{mmol})$ in anhydrous THF (10 $\mathrm{mL}$ ) was added to a stirred suspension of $\mathrm{NaH}$ (abs. $60 \%$, $407 \mathrm{mg}, 10.1 \mathrm{mmol})$ in anhydrous THF $(10 \mathrm{~mL})$ at $0{ }^{\circ} \mathrm{C}$ under argon. The mixture was stirred at $0{ }^{\circ} \mathrm{C}$ for $20 \mathrm{~min}$, and then benzyl bromide $(1.27 \mathrm{~mL}, 10.6 \mathrm{mmol})$ was added to the solution. After being stirred at $0{ }^{\circ} \mathrm{C}$ for $20 \mathrm{~min}$, the reaction mixture was allowed to warm to room temperature and then stirred for $5 \mathrm{~h}$. The reaction mixture was concentrated in vacuo and was treated with $1 \mathrm{~N} \mathrm{HCl}(10 \mathrm{~mL})$ and then extracted with $\mathrm{CHCl}_{3}(50 \mathrm{~mL} \times 3)$. The extract was dried over anhydrous $\mathrm{MgSO}_{4}$, filtered, and concentrated in vacuo. The oily residue was purified by silica gel column chromatography [Silica Gel 60N, $n$-hexane-AcOEt (7:1)] to afford 3a $(3.19 \mathrm{~g}, 82 \%)$ as colorless oil.

\section{Diethyl 2-Benzyl-2-(benzyloxycarbonylamino)malonate (3a) \\ Colorless oil; ${ }^{1} \mathrm{H}$ NMR (400 $\left.\mathrm{MHz}, \mathrm{CDCl}_{3}\right) \delta$ 1.11-1.32 $(\mathrm{m}, 6 \mathrm{H}), 3.61(\mathrm{~s}, 2 \mathrm{H}), 4.04-4.32(\mathrm{~m}, 4 \mathrm{H}), 5.15(\mathrm{~s}, 2 \mathrm{H}), 5.96$ $(\mathrm{s}, 1 \mathrm{H}), 6.85-6.99(\mathrm{~m}, 2 \mathrm{H}), 7.08-7.46(\mathrm{~m}, 8 \mathrm{H}) ;{ }^{13} \mathrm{C} \mathrm{NMR}$}


$\left(100 \mathrm{MHz}, \mathrm{CDCl}_{3}\right) \delta 13.9,38.1,62.5,66.8,67.3,127.0$, 128.0, 128.1, 128.3, 128.4, 129.8, 134.7, 136.2, 154.1, 167.1; IR (neat) 3426, 2983, 1742, 1495, 1370, 1212, 1074, $749 \mathrm{~cm}^{-}$

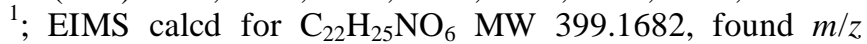
$399.1662\left(\mathrm{M}^{+}\right)$; Anal. Calcd for $\mathrm{C}_{22} \mathrm{H}_{25} \mathrm{NO}_{6}$ : C, 66.15; $\mathrm{H}$, 6.31 ; N, 3.51. Found: C, 65.91; H, 6.27; N, 3.48\%.

Diethyl 2-(Benzyloxycarbonylamino)-2-methylmalonate (3b)

Colorless oil; ${ }^{1} \mathrm{H}$ NMR (400 MHz, $\left.\mathrm{CDCl}_{3}\right) \delta$ 1.09-1.43 (m, 6H), 1.77 (s, 3H), 4.02-4.40 (m, 4H), 5.10 (s, 2H), 6.20 $(\mathrm{s}, 1 \mathrm{H}), 7.21-7.62(\mathrm{~m}, 5 \mathrm{H}) ;{ }^{13} \mathrm{C} \mathrm{NMR}\left(100 \mathrm{MHz}, \mathrm{CDCl}_{3}\right) \delta$ $13.9,21.2,62.5,63.1,66.8,127.9,128.0,128.3,136.1$, 154.2, 168.4; IR (neat) 3423, 2985, 1732, 1499, 1376, 1206,

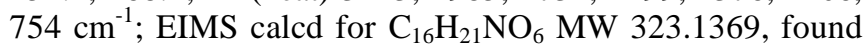
$m / z 323.1372\left(\mathrm{M}^{+}\right)$; Anal. Calcd for $\mathrm{C}_{16} \mathrm{H}_{21} \mathrm{NO}_{6}$ : C, 59.43; $\mathrm{H}$, 6.55; N, 4.33. Found: C, 59.03; H, 6.52; N, $4.51 \%$.

\section{Diethyl 2-Allyl-2-(benzyloxycarbonylamino)malonate (3c)}

Colorless oil; ${ }^{1} \mathrm{H}$ NMR (400 MHz, $\left.\mathrm{CDCl}_{3}\right) \delta$ 1.11-1.29 $(\mathrm{m}, 6 \mathrm{H}), 3.06(\mathrm{~d}, J=6.8 \mathrm{~Hz}, 2 \mathrm{H}), 3.99-4.35(\mathrm{~m}, 4 \mathrm{H}), 4.97-$ $5.25(\mathrm{~m}, 4 \mathrm{H}), 5.51-5.68(\mathrm{~m}, 1 \mathrm{H}), 6.14(\mathrm{~s}, 1 \mathrm{H}), 7.20-7.44(\mathrm{~m}$. $5 \mathrm{H}) ;{ }^{13} \mathrm{C}$ NMR $\left(100 \mathrm{MHz}, \mathrm{CDCl}_{3}\right) \delta 13.9,37.3,62.5,66.3$, 66.7, 119.7, 127.9, 128.0, 128.3, 131.0, 136.1, 154.1, 167.3; IR (neat) 3067, 1741, 1643, 1495, 1227, 1138, 1029, 927, $741 \mathrm{~cm}^{-1}$; EIMS calcd for $\mathrm{C}_{18} \mathrm{H}_{23} \mathrm{NO}_{6} \mathrm{MW} 349.1525$, found $m / z$ 349.1531 $\left(\mathrm{M}^{+}\right)$; Anal. Calcd for $\mathrm{C}_{18} \mathrm{H}_{23} \mathrm{NO}_{6}: \mathrm{C}, 61.88 ; \mathrm{H}$, $6.64 ; \mathrm{N}, 4.01$. Found: C, 61.55; H, 6.52; N, 3.95\%.

\section{Diethyl 2-(Benzyloxycarbonylamino)-2-hexylmalonate (3d)}

Colorless oil; ${ }^{1} \mathrm{H}$ NMR (400 MHz, $\left.\mathrm{CDCl}_{3}\right) \delta$ 0.77-0.92 (m, 3H), 1.02-1.39 (m, 14H), 2.16-2.41 (m, 2H), 3.96-4.36 $(\mathrm{m}, 4 \mathrm{H}), 5.09(\mathrm{~s}, 2 \mathrm{H}), 6.17(\mathrm{~s}, 1 \mathrm{H}), 7.17-7.61(\mathrm{~m}, 5 \mathrm{H}) ;{ }^{13} \mathrm{C}$ NMR $\left(100 \mathrm{MHz}, \mathrm{CDCl}_{3}\right) \delta 13.9,14.0,22.5,23.3,28.9,31.5$, 32.4, 62.4, 66.7, 127.9, 128.0, 128.3, 136.2, 154.1, 167.9; IR (neat) 3425, 2930, 2860, 1732, 1495, 1370, 1251, 1201, 739

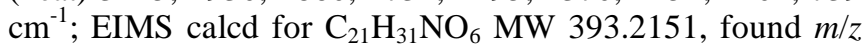
393.2149 $\left(\mathrm{M}^{+}\right)$; Anal. Calcd for $\mathrm{C}_{21} \mathrm{H}_{31} \mathrm{NO}_{6}$ : C, 64.10; $\mathrm{H}$, 7.94; N, 3.56. Found: C, 64.03; H, 7.89; N, 3.78\%.

\section{Reduction of Diethyl 2-Benzyl-2-(benzyloxycarbonyl- amino)malonate (3a) with $\mathrm{LiBH}_{4}$}

To a solution of $\mathbf{3 a}(510 \mathrm{mg}, 1.28 \mathrm{mmol})$ in anhydrous THF (10 mL) was added $\mathrm{LiBH}_{4}(112 \mathrm{mg}, 5.12 \mathrm{mmol})$ at $0{ }^{\circ} \mathrm{C}$ under argon. After being stirred at $0{ }^{\circ} \mathrm{C}$ for $1 \mathrm{~h}$, the reaction mixture was allowed to warm to room temperature and then stirred for $20 \mathrm{~h}$. The reaction mixture was concentrated in vacuo and was treated with $1 \mathrm{~N} \mathrm{HCl}(10 \mathrm{~mL})$ and then extracted with $\mathrm{CHCl}_{3}(30 \mathrm{~mL}$ x 6$)$. The extract was dried over anhydrous $\mathrm{MgSO}_{4}$, filtered, and concentrated in vacuo. The oily residue was purified by silica gel column chromatography [Silica Gel 60N, $n$-hexane-AcOEt (1:1)] to afford 4a (236 mg, 59\%).

\section{Benzyl 2-Benzyl-1,3-dihydroxypropan-2-ylcarbamate (4a)}

Colorless needles $\left(\mathrm{CHCl}_{3}-n\right.$-hexane), mp 81-82 ${ }^{\circ} \mathrm{C} ;{ }^{1} \mathrm{H}$ $\mathrm{NMR}\left(400 \mathrm{MHz}, \mathrm{CDCl}_{3}\right) \delta 2.92(\mathrm{~s}, 2 \mathrm{H}), 3.10-3.21(\mathrm{~m}, 2 \mathrm{H})$, $3.61(\mathrm{dd}, J=7.1,11.7 \mathrm{~Hz}, 2 \mathrm{H}), 3.80(\mathrm{dd}, J=5.9,11.7 \mathrm{~Hz}, 2 \mathrm{H})$, $5.10(\mathrm{~s}, 2 \mathrm{H}), 5.16(\mathrm{~s}, 1 \mathrm{H}), 7.15-7.21(\mathrm{~m}, 2 \mathrm{H}), 7.21-7.31(\mathrm{~m}$, $3 \mathrm{H}), 7.31-7.45(\mathrm{~m}, 5 \mathrm{H}) ;{ }^{13} \mathrm{C} \mathrm{NMR}\left(75 \mathrm{MHz}, \mathrm{CDCl}_{3}\right) \delta 37.9$, $59.7,65.3,66.9,126.9,128.1,128.3,128.5,128.6,130.3$, 135.6, 136.1, 156.5; IR (KBr) 3382, 3299, 1695, 1542, 1523, 1454, 1282, 1241, $1027 \mathrm{~cm}^{-1}$; EIMS calcd for $\mathrm{C}_{18} \mathrm{H}_{22} \mathrm{NO}_{4}$
MW 316.1549, found $m / z$ 316.1531 $\left(\mathrm{M}^{+}+\mathrm{H}\right)$; Anal. Calcd for $\mathrm{C}_{18} \mathrm{H}_{21} \mathrm{NO}_{4}$ : C, 68.55; H, 6.71; N, 4.44. Found: C, 68.36; $\mathrm{H}, 6.75 ; \mathrm{N}, 4.44 \%$.

\section{Benzyl 1,3-Dihydroxy-2-methylpropan-2-ylcarbamate (4b)}

White powder $\left(\mathrm{CHCl}_{3}-n\right.$-hexane $), \mathrm{mp} 79-80{ }^{\circ} \mathrm{C} ;{ }^{1} \mathrm{H}$ NMR (400 MHz, $\left.\mathrm{CDCl}_{3}\right) \delta 1.20(\mathrm{~s}, 3 \mathrm{H}), 3.09-3.24(\mathrm{~m}, 2 \mathrm{H})$, $3.66(\mathrm{dd}, J=6.6,11.5 \mathrm{~Hz}, 2 \mathrm{H}), 3.80(\mathrm{dd}, J=5.9,11.5 \mathrm{~Hz}, 2 \mathrm{H})$, $5.08(\mathrm{~s}, 2 \mathrm{H}), 5.26(\mathrm{~s}, 1 \mathrm{H}), 7.29-7.43(\mathrm{~m}, 5 \mathrm{H}) ;{ }^{13} \mathrm{C}$ NMR $(75$ $\left.\mathrm{MHz}, \mathrm{CDCl}_{3}\right) \delta 20.0,57.2,66.8,67.7,128.1,128.2,128.6$, 136.1, 156.4; IR (KBr) 3274, 3087, 1691, 1673, 1558, 1455, 1288, 1267, $1253 \mathrm{~cm}^{-1}$; EIMS calcd for $\mathrm{C}_{12} \mathrm{H}_{18} \mathrm{NO}_{4} \mathrm{MW}$ 240.1236, found $m / z 240.1253\left(\mathrm{M}^{+}+\mathrm{H}\right)$; Anal. Calcd for $\mathrm{C}_{12} \mathrm{H}_{17} \mathrm{NO}_{4}$ : C, 60.24; H, 7.16; N, 5.85. Found: C, 60.07; H, $7.14 ; \mathrm{N}, 5.83 \%$.

\section{Benzyl 1-Hydroxy-2-(hydroxymethyl)pent-4-en-2-ylcarba- mate $(4 c)$}

Colorless oil; ${ }^{1} \mathrm{H}$ NMR (400 MHz, $\left.\mathrm{CDCl}_{3}\right) \delta 2.35(\mathrm{~d}$, $J=7.6 \mathrm{~Hz}, 2 \mathrm{H}), 3.36-3.54(\mathrm{~m}, 2 \mathrm{H}), 3.61(\mathrm{dd}, J=7.1,11.7 \mathrm{~Hz}$, 2H), 3.83 (dd, J=5.1, $11.7 \mathrm{~Hz}, 2 \mathrm{H}), 5.07$ (s, 2H), 5.11-5.24 $(\mathrm{m}, 2 \mathrm{H}), 5.29$ (s, 1H), 5.72-5.88 (m, 1H), 7.29-7.45 (m, 5H); ${ }^{13} \mathrm{C}$ NMR $\left(75 \mathrm{MHz}, \mathrm{CDCl}_{3}\right) \delta 37.4,59.0,65.9,67.0,120.0$, $128.1,128.3,128.6,132.2,136.0,156.6$; IR (neat) 3396, 2948, 2888, 1697, 1513, 1455, 1240, $1039 \mathrm{~cm}^{-1}$; FABMS calcd for $\mathrm{C}_{14} \mathrm{H}_{20} \mathrm{NO}_{4}$ MW 266.1392, found $\mathrm{m} / \mathrm{z} 266.1418$ $\left(\mathrm{M}^{+}+\mathrm{H}\right)$.

\section{Benzyl 1-Hydroxy-2-(hydroxymethyl)octan-2-ylcarbamate (4d)}

Colorless plates $\left(\mathrm{CHCl}_{3}-n\right.$-hexane $), \operatorname{mp} 69-70{ }^{\circ} \mathrm{C} ;{ }^{1} \mathrm{H}$ NMR $\left(400 \mathrm{MHz}, \mathrm{CDCl}_{3}\right) \delta 0.88(\mathrm{t}, J=6.8 \mathrm{~Hz}, 3 \mathrm{H}), 1.17-1.36$ $(\mathrm{m}, 8 \mathrm{H}), 1.48-1.61(\mathrm{~m}, 2 \mathrm{H}), 3.27-3.42(\mathrm{~m}, 2 \mathrm{H}), 3.62(\mathrm{dd}$, $J=6.8,11.5 \mathrm{~Hz}, 2 \mathrm{H}), 3.86(\mathrm{dd}, J=5.9,11.5 \mathrm{~Hz}, 2 \mathrm{H}), 5.07$ (s, $2 \mathrm{H}), 5.19(\mathrm{~s}, 1 \mathrm{H}), 7.28-7.44(\mathrm{~m}, 5 \mathrm{H}) ;{ }^{13} \mathrm{C} \mathrm{NMR}(75 \mathrm{MHz}$, $\left.\mathrm{CDCl}_{3}\right) \delta 14.0,22.5,22.9,29.7,31.6,33.2,59.5,66.3,66.8$, 128.1, 128.2, 128.5, 136.1, 156.6; IR (KBr) 3280, 2954, 2937, 2911, 2883, 2856, 1673, 1467, 1428, $1336 \mathrm{~cm}^{-1}$; EIMS calcd for $\mathrm{C}_{17} \mathrm{H}_{28} \mathrm{NO}_{4}$ MW 310.2018, found $\mathrm{m} / \mathrm{z} 310.2004$ $\left(\mathrm{M}^{+}+\mathrm{H}\right)$; Anal. Calcd for $\mathrm{C}_{17} \mathrm{H}_{27} \mathrm{NO}_{4}$ : C, 65.99; $\mathrm{H}, 8.80 ; \mathrm{N}$, 4.53. Found: C, 65.79; H, 8.78; N, 4.50\%.

\section{Enzyme-Catalyzed Acetylation of Benzyl 2-Benzyl-1,3- dihydroxypropan-2-ylcarbamate (4a) with Immobilized Lipoprotein Lipase}

Immobilized LPL from Pseudomonas sp. (Toyobo; LIP$301,50 \mathrm{mg}, 29.5$ units) and vinyl acetate $(196 \mu \mathrm{L}, 2.292$ mmol) were added to a stirred solution of $\mathbf{4 a}(180 \mathrm{mg}, 0.573$ $\mathrm{mmol})$ in TBME $(10 \mathrm{~mL})$ at room temperature. After being stirred at room temperature for $2 \mathrm{~h}$, the reaction mixture was filtered, and concentrated in vacuo. The oily residue was purified by silica gel column chromatography [Silica Gel $60 \mathrm{~N}, n$-hexane-AcOEt $(2: 1)]$ to afford $(R)-5 \mathbf{a}(193 \mathrm{mg}$, $94 \% .97 \%$ ee) as a white solid. The ee of $\mathbf{5 a}$ was determined by HPLC analysis (CHIRALCEL OJ-H, n-hexane/2propanol $=5 / 1,1.0 \mathrm{ml} / \mathrm{min}, 254 \mathrm{~nm}$ ).

\section{(R)-2-Benzyl-2-(benzyloxycarbonylamino)-3-hydroxypropyl Acetate [(R)-5a]}

$100 \%$ ee; Colorless needles (AcOEt- $n$-hexane), mp 66.5-67 ${ }^{\circ} \mathrm{C} ;[\alpha]_{\mathrm{D}}{ }^{24}+4.8$ (c 1.02 in $\left.\mathrm{CHCl}_{3}\right) ;{ }^{1} \mathrm{H}$ NMR $(400$ $\left.\mathrm{MHz}, \mathrm{CDCl}_{3}\right) \delta 2.09$ (s, 3H), 2.95 (d, J=13.6 Hz, 1H), 3.15 
(d, $J=13.4 \mathrm{~Hz}, 1 \mathrm{H}), 3.44-3.57(\mathrm{~m}, 1 \mathrm{H}), 3.61$ (dd, $J=7.1,11.9$ $\mathrm{Hz}, 1 \mathrm{H}), 3.69$ (dd, J=6.6, $11.5 \mathrm{~Hz}, 1 \mathrm{H}), 4.11$ (d, $J=11.5 \mathrm{~Hz}$, $1 \mathrm{H}), 4.24(\mathrm{~d}, J=11.5 \mathrm{~Hz}, 1 \mathrm{H}), 4.93(\mathrm{~s}, 1 \mathrm{H}), 5.11(\mathrm{~s}, 2 \mathrm{H})$, 7.03-7.15 (m, 2H), 7.16-7.30 (m, 3H), 7.30-7.48 (m, 5H); ${ }^{13} \mathrm{C}$ NMR $\left(75 \mathrm{MHz}, \mathrm{CDCl}_{3}\right) \delta 20.8,37.1,59.1,63.7,64.3$, $66.7,126.8,128.21,128.24,128.4,128.5,130.4,135.4$, 136.2, 155.7, 171.1; IR (KBr) 3448, 2362, 1708, 1558, 1454, 1376, 1234, 1081, $1039 \mathrm{~cm}^{-1}$; ESIMS calcd for $\mathrm{C}_{20} \mathrm{H}_{23} \mathrm{NO}_{5} \mathrm{Na} \mathrm{MW} 380.1474$, found $\mathrm{m} / \mathrm{z} 380.1475\left(\mathrm{M}^{+}+\right.$ $\mathrm{Na}$ ); Anal. Calcd for $\mathrm{C}_{20} \mathrm{H}_{23} \mathrm{NO}_{5}$ : C, 67.21; H, 6.49; N, 3.92. Found: C, 67.11; H, 6.52; N, 3.95\%.

(R)-2-(Benzyloxycarbonylamino)-3-hydroxy-2-methylpropyl Acetate [(R)-5b]

92\% ee; Colorless oil; $[\alpha]_{\mathrm{D}}{ }^{30}-7.2$ (c 1.63 in $\left.\mathrm{CHCl}_{3}\right) ;{ }^{1} \mathrm{H}$ NMR (400 MHz, $\left.\mathrm{CDCl}_{3}\right) \delta 1.30$ (s, 3H), 2.09 (s, 3H), 3.413.51 (brs, $1 \mathrm{H}), 3.58-3.68(\mathrm{~m}, 2 \mathrm{H}), 4.21(\mathrm{~d}, J=11.2 \mathrm{~Hz}, 1 \mathrm{H})$, 4.25 (d, $J=11.2 \mathrm{~Hz}, 1 \mathrm{H}), 5.07$ (s, 2H), 5.15 (s, 1H), 7.31-7.37 $(\mathrm{m}, 5 \mathrm{H}) ;{ }^{13} \mathrm{C}$ NMR $\left(100 \mathrm{MHz}, \mathrm{CDCl}_{3}\right) \delta 19.7,20.8,56.4$, 66.1, 66.4, 66.7, 128.0, 128.1, 128.4, 136.0, 155.6, 171.0; IR (neat) 3359, 2946, 1714, 1536, 1455, 1375, 1241, $1045 \mathrm{~cm}^{-1}$;

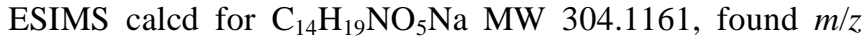
$304.1167\left(\mathrm{M}^{+}+\mathrm{Na}\right)$.

\section{2-(Benzyloxycarbonylamino)-2-(hydroxymethyl)pent-4-enyl Acetate (5c)}

98\% ee; Colorless oil; $[\alpha]_{\mathrm{D}}{ }^{27}-15.0$ (c 1.19 in $\left.\mathrm{CHCl}_{3}\right) ;{ }^{1} \mathrm{H}$ NMR $\left(400 \mathrm{MHz}, \mathrm{CDCl}_{3}\right) \delta 2.08(\mathrm{~s}, 3 \mathrm{H}), 2.34(\mathrm{dd}, J=8.0$, $14.1 \mathrm{~Hz}, 1 \mathrm{H}), 2.57(\mathrm{dd}, J=6.8,13.9 \mathrm{~Hz}, 1 \mathrm{H}), 3.52-3.80(\mathrm{~m}$, $3 \mathrm{H}), 4.16(\mathrm{~d}, J=11.4 \mathrm{~Hz}, 1 \mathrm{H}), 4.30$ (d, $J=11.4 \mathrm{~Hz}, 1 \mathrm{H}), 5.07$ (s, 2H), $5.11(\mathrm{~s}, 1 \mathrm{H}), 5.13-5.21(\mathrm{~m}, 2 \mathrm{H}), 5.68-5.87(\mathrm{~m}, 1 \mathrm{H})$, 7.29-7.41 (m, 5H); ${ }^{13} \mathrm{C} \mathrm{NMR}\left(100 \mathrm{MHz}, \mathrm{CDCl}_{3}\right) \delta 20.7$, $36.8,58.2,64.5,64.6,66.6,119.6,127.9,128.0,128.3$, 131.7, 135.9, 155.6, 170.8; IR (neat) 3407, 3068, 3033, 3008, 2956, 2894, 1729, 1714, 1641, 1538, 1515, 1506, 1455, 1380, 1236, $1041 \mathrm{~cm}^{-1}$; ESIMS calcd for $\mathrm{C}_{16} \mathrm{H}_{21} \mathrm{NO}_{5} \mathrm{Na}$ MW 330.1317, found $\mathrm{m} / \mathrm{z} 330.1302\left(\mathrm{M}^{+}+\right.$ $\mathrm{Na})$.

\section{2-(Benzyloxycarbonylamino)-2-(hydroxymethyl)octyl Ace- tate (5d)}

96\% ee; Colorless oil; $[\alpha]_{\mathrm{D}}{ }^{26}-7.6\left(c 0.97\right.$ in $\left.\mathrm{CHCl}_{3}\right) ;{ }^{1} \mathrm{H}$ NMR (400 MHz, $\left.\mathrm{CDCl}_{3}\right) \delta 0.88(\mathrm{t}, J=7.1 \mathrm{~Hz}, 3 \mathrm{H}), 1.16-1.35$ $(\mathrm{m}, 8 \mathrm{H}), 1.53-1.64(\mathrm{~m}, 1 \mathrm{H}), 1.67-1.80(\mathrm{~m}, 1 \mathrm{H}), 2.08(\mathrm{~s}, 3 \mathrm{H})$, 3.54-3.77 (m, 3H), 4.15 (d, J=11.4 Hz, 1H), 4.29 (d, J=11.4 $\mathrm{Hz}, 1 \mathrm{H}), 5.04$ (s, 1H), 5.07 (s, 2H), 7.29-7.42 (m, 5H); ${ }^{13} \mathrm{C}$ NMR (100 MHz, $\left.\mathrm{CDCl}_{3}\right) \delta 14.0,20.7,22.5,22.8,29.6,31.5$, $32.5,58.7,64.5,65.1,66.6,127.9,128.0,128.3,136.0$, 155.7, 170.9; IR (neat) 3353, 2954, 2929, 2859, 1729, 1536, 1455, 1378, 1238, $1041 \mathrm{~cm}^{-1}$; ESIMS calcd for $\mathrm{C}_{19} \mathrm{H}_{29} \mathrm{NO}_{5} \mathrm{Na}$ MW 374.1943, found $\mathrm{m} / z$ 374.1949 $\left(\mathrm{M}^{+}+\right.$ $\mathrm{Na})$.

\section{2-(Benzyloxycarbonylamino)-3-hydroxy-2-methylpropyl 2- Chloroacetate (5ba)}

$44 \%$ ee; Colorless oil; $[\alpha]_{\mathrm{D}}{ }^{27}-2.4\left(c 0.85\right.$ in $\left.\mathrm{CHCl}_{3}\right) ;{ }^{1} \mathrm{H}$ NMR (400 MHz, $\left.\mathrm{CDCl}_{3}\right) \delta 1.29(\mathrm{~s}, 3 \mathrm{H}), 3.25-3.47(\mathrm{~m}, 1 \mathrm{H})$, 3.61-3.75 (m, 2H), $4.07(\mathrm{~s}, 2 \mathrm{H}), 4.35(\mathrm{~d}, J=11.0 \mathrm{~Hz}, 1 \mathrm{H})$, $4.40(\mathrm{~d}, J=11.0 \mathrm{~Hz}, 1 \mathrm{H}), 5.07$ (s, 2H), 5.12 (s, 1H), 7.29-7.41 $(\mathrm{m}, 5 \mathrm{H}) ;{ }^{13} \mathrm{C}$ NMR $\left(100 \mathrm{MHz}, \mathrm{CDCl}_{3}\right) \delta 19.9,40.6,56.2$, $66.3,66.8,67.3,127.9,128.1,128.4,135.8,155.7,167.1$; IR (neat) 3403, 3066, 3033, 2954, 2890, 2360, 1729, 1712,
1529, 1454, 1411, 1371, 1249, 1191, $1074 \mathrm{~cm}^{-1}$; ESIMS calcd for $\mathrm{C}_{14} \mathrm{H}_{18} \mathrm{ClNO}_{5} \mathrm{Na}$ MW 338.0771, found $\mathrm{m} / \mathrm{z}$ $338.0770\left(\mathrm{M}^{+}+\mathrm{Na}\right)$.

\section{2-(Benzyloxycarbonylamino)-3-hydroxy-2-methylpropyl Butyrate (5bb)}

$83 \%$ ee; Colorless oil; $[\alpha]_{\mathrm{D}}{ }^{27}-6.4\left(c 0.73\right.$ in $\left.\mathrm{CHCl}_{3}\right) ;{ }^{1} \mathrm{H}$ NMR (400 MHz, $\left.\mathrm{CDCl}_{3}\right) \delta 0.95(\mathrm{t}, J=7.3 \mathrm{~Hz}, 3 \mathrm{H}), 1.30(\mathrm{~s}$, $3 \mathrm{H}), 1.54-1.72(\mathrm{~m}, 2 \mathrm{H}), 2.32(\mathrm{t}, J=7.3 \mathrm{~Hz}, 2 \mathrm{H}), 3.49$ (brs, $1 \mathrm{H}), 3.59$ (dd, $J=6.1,12.0 \mathrm{~Hz}, 1 \mathrm{H}), 3.65(\mathrm{dd}, J=6.1,11.7 \mathrm{~Hz}$, $1 \mathrm{H}), 4.21(\mathrm{~d}, J=11.2 \mathrm{~Hz}, 1 \mathrm{H}), 4.27$ (d, $J=11.5 \mathrm{~Hz}, 1 \mathrm{H}), 5.07$ $(\mathrm{s}, 2 \mathrm{H}), 5.15(\mathrm{~s}, 1 \mathrm{H}), 7.27-7.41(\mathrm{~m}, 5 \mathrm{H}) ;{ }^{13} \mathrm{C}$ NMR $(100$ $\left.\mathrm{MHz}, \mathrm{CDCl}_{3}\right) \delta 13.6,18.3,19.6,36.0,56.3,65.7,66.3,66.6$, $127.9,128.0,128.3,136.0,155.6,173.6$; IR (neat) 3361, 2965, 2877, 1727, 1714, 1546, 1531, 1515, 1504, 1454, 1380, 1249, 1182, $1074 \mathrm{~cm}^{-1}$; ESIMS calcd for $\mathrm{C}_{16} \mathrm{H}_{23} \mathrm{NO}_{5} \mathrm{Na} \mathrm{MW} 332.1474$, found $\mathrm{m} / \mathrm{z} 332.1451\left(\mathrm{M}^{+}+\right.$ $\mathrm{Na}$ ).

\section{2-(Benzyloxycarbonylamino)-3-hydroxy-2-methylpropyl Benzoate (5bc)}

$79 \%$ ee; Colorless oil; $[\alpha]_{\mathrm{D}}{ }^{24}-10.7\left(c 0.58\right.$ in $\left.\mathrm{CHCl}_{3}\right) ;{ }^{1} \mathrm{H}$ NMR (400 MHz, $\left.\mathrm{CDCl}_{3}\right) \delta 1.39(\mathrm{~s}, 3 \mathrm{H}), 3.45-3.86(\mathrm{~m}, 3 \mathrm{H})$, 4.45-4.75 (m, 2H), 5.02-5.16 (m, 2H), $5.27(\mathrm{~s}, 1 \mathrm{H}), 7.27-$ $7.53(\mathrm{~m}, 7 \mathrm{H}), 7.54-7.65(\mathrm{~m}, 1 \mathrm{H}), 7.99-8.08(\mathrm{~m}, 2 \mathrm{H}) ;{ }^{13} \mathrm{C}$ NMR $\left(75 \mathrm{MHz}, \mathrm{CDCl}_{3}\right) \delta 19.8,56.7,66.3,66.5,66.8,128.1$, $128.2,128.52,128.57,129.4,129,7,133.4,136.1,155.8$, 166.8; IR (neat) 3357, 3066, 3033, 2944, 2890, 1714, 1536, 1452, 1371, 1315, 1276, 1116, $1070 \mathrm{~cm}^{-1}$; ESIMS calcd for $\mathrm{C}_{19} \mathrm{H}_{21} \mathrm{NO}_{5} \mathrm{Na} \mathrm{MW} 366.1317$, found $\mathrm{m} / \mathrm{z} 366.1284\left(\mathrm{M}^{+}+\right.$ $\mathrm{Na}$; Anal. Calcd for $\mathrm{C}_{19} \mathrm{H}_{21} \mathrm{NO}_{5}$ : C, 66.46; H, 6.16; N, 4.08. Found: C, 66.22; H, 6.42; N, 4.04\%.

\section{2-(Benzyloxycarbonylamino)-3-hydroxy-2-methylpropyl Pivalate (5bd)}

$59 \%$ ee; Colorless oil; $[\alpha]_{\mathrm{D}}{ }^{27}-4.4\left(c 0.54\right.$ in $\left.\mathrm{CHCl}_{3}\right) ;{ }^{1} \mathrm{H}$ NMR (400 MHz, $\left.\mathrm{CDCl}_{3}\right) \delta 1.20(\mathrm{~s}, 9 \mathrm{H}), 1.30$ (s, 3H), 3.36$3.67(\mathrm{~m}, 3 \mathrm{H}), 4.19(\mathrm{~d}, J=11.2 \mathrm{~Hz}, 1 \mathrm{H}), 4.26(\mathrm{~d}, J=11.2 \mathrm{~Hz}$, 1H), 5.06 (s, 2H), $5.16(\mathrm{~s}, 1 \mathrm{H}), 7.27-7.41(\mathrm{~m}, 5 \mathrm{H}) ;{ }^{13} \mathrm{C} \mathrm{NMR}$ $\left(75 \mathrm{MHz} \mathrm{CDCl}_{3}\right) \delta 19.6,27.1,38.9,56.6,65.6,66.5,66.7$, $128.1,128.2,128.5,136.1,155.7,178.8$; IR (neat) 3357, 2973, 1731, 1716, 1558, 1540, 1521, 1508, $1455 \mathrm{~cm}^{-1}$; ESIMS calcd for $\mathrm{C}_{17} \mathrm{H}_{26} \mathrm{NO}_{5}$ MW 324.1811, found $\mathrm{m} / \mathrm{z}$ 324.1811 $\left(\mathrm{M}^{+}+\mathrm{H}\right)$.

\section{(S)-2-Benzyl-2-(benzyloxycarbonylamino)-3-oxopropyl Acetate $[(S)-6 a]$}

To a solution of $(S)-5 a$ (351 $\mathrm{mg}, 0.98 \mathrm{mmol}, 100 \%$ ee) and TEA (1.37 mL, $9.82 \mathrm{mmol})$ in anhydrous DMSO (10 $\mathrm{mL})$ was added $\mathrm{SO}_{3}$-pyridine $(1.25 \mathrm{~g}, 7.86 \mathrm{mmol})$ at room temperature under argon. After being stirred at room temperature for $30 \mathrm{~min}$, the reaction mixture was treated with 1 $\mathrm{N} \mathrm{HCl}(5 \mathrm{~mL})$ and then extracted with $\mathrm{CHCl}_{3}(20 \mathrm{~mL} \times 3)$. The extract was dried over anhydrous $\mathrm{MgSO}_{4}$, filtered, and concentrated in vacuo. The oily residue was purified by silica gel column chromatography [Silica Gel $60 \mathrm{~N}, n$-hexaneAcOEt (3:1)] to afford (S)-6a (345 mg, 99\%) as colorless oil. $[\alpha]_{\mathrm{D}}{ }^{28}+1.5($ c 1.59 in $\mathrm{MeOH}) ;{ }^{1} \mathrm{H}$ NMR $\left(400 \mathrm{MHz}, \mathrm{CDCl}_{3}\right)$ $\delta 2.01(\mathrm{~s}, 3 \mathrm{H}), 3.16(\mathrm{~d}, J=13.9 \mathrm{~Hz}, 1 \mathrm{H}), 3.38$ (d, $J=13.9 \mathrm{~Hz}$, $1 \mathrm{H}), 4.47(\mathrm{~d}, J=11.7 \mathrm{~Hz}, 1 \mathrm{H}), 4.66(\mathrm{~d}, J=11.7 \mathrm{~Hz}, 1 \mathrm{H}), 5.10$ $(\mathrm{d}, J=12.2 \mathrm{~Hz}, 1 \mathrm{H}), 5.19(\mathrm{~d}, J=12.2 \mathrm{~Hz}, 1 \mathrm{H}), 5.47(\mathrm{~s}, 1 \mathrm{H})$, 6.89-7.02 (m, 2H), 7.11-7.52 (m, 8H), $9.53(\mathrm{~s}, 1 \mathrm{H}) ;{ }^{13} \mathrm{C}$ 
NMR (100 MHz, $\left.\mathrm{CDCl}_{3}\right) \delta 20.5,35.7,62.9,66.1,66.7$, 127.1, 128.0, 128.1, 128.3, 129.8, 133.4, 136.0, 154.7, 170.2, 197.1; IR (neat) 3403, 3340, 3031, 2360, 1957, 1745, 1716, 1500, 1455, 1376, 1317, 1226, 1076, $1043 \mathrm{~cm}^{-1}$; ESIMS calcd for $\mathrm{C}_{20} \mathrm{H}_{21} \mathrm{NO}_{5} \mathrm{Na}$ MW 378.1317, found $\mathrm{m} / z 378.1313$ $\left(\mathrm{M}^{+}+\mathrm{Na}\right)$.

\section{(S)-2-(Benzyloxycarbonylamino)-2-methyl-3-oxopropyl Acetate [(S)-6b]}

Colorless oil. $[\alpha]_{\mathrm{D}}{ }^{26}-12.7$ (c 1.05 in $\left.\mathrm{MeOH}\right) ;{ }^{1} \mathrm{H}$ NMR $\left(400 \mathrm{MHz}, \mathrm{CDCl}_{3}\right) \delta 1.45(\mathrm{~s}, 3 \mathrm{H}), 2.04(\mathrm{~s}, 3 \mathrm{H}), 4.38(\mathrm{~d}$, $J=11.7 \mathrm{~Hz}, 1 \mathrm{H}), 4.51(\mathrm{~d}, J=11.7 \mathrm{~Hz}, 1 \mathrm{H}), 5.11(\mathrm{~s}, 2 \mathrm{H}), 5.61$ $(\mathrm{s}, 1 \mathrm{H}), 7.32-7.37(\mathrm{~m}, 5 \mathrm{H}), 9.44(\mathrm{~s}, 1 \mathrm{H}) ;{ }^{13} \mathrm{C}$ NMR $(100$ $\left.\mathrm{MHz}, \mathrm{CDCl}_{3}\right) \delta 17.4,20.6,62.2,64.1,66.9,128.0,128.1$, 128.4, 135.8, 154.8, 170.4, 197.7; IR (neat) 3342, 3033, 1743, 1521, 1455, 1375, 1238, 1076, 1049, 916, 742, 698 $\mathrm{cm}^{-1}$; ESIMS calcd for $\mathrm{C}_{14} \mathrm{H}_{17} \mathrm{NO}_{5} \mathrm{Na} \mathrm{MW} 302.1104$, found $m / z, 302.0989\left(\mathrm{M}^{+}+\mathrm{Na}\right)$.

(S)-3-Acetoxy-2-benzyl-2-(benzyloxycarbonylamino)propanoic Acid $[(S)-7 a]$

To a solution of $(S)-6 \mathbf{a}(308 \mathrm{mg}, 0.867 \mathrm{mmol}), 2$-methyl2-butene $(367 \mu \mathrm{L}, 3.47 \mathrm{mmol})$ and $\mathrm{NaH}_{2} \mathrm{PO}_{4}(135 \mathrm{mg}, 0.867$ mmol) in $\mathrm{H}_{2} \mathrm{O}$-tert-BuOH $(1: 4,10 \mathrm{~mL})$ was added $\mathrm{NaClO}_{2}$ (235 $\mathrm{mg}, 2.60 \mathrm{mmol}$ ) at room temperature. After being stirred at room temperature for $1 \mathrm{~h}$, the reaction mixture was treated with $1 \mathrm{~N} \mathrm{HCl}(5 \mathrm{~mL})$ and then extracted with $\mathrm{CHCl}_{3}$ (20 mL x 6). The extract was dried over anhydrous $\mathrm{MgSO}_{4}$, filtered, and concentrated in vacuo. The oily residue was purified by silica gel column chromatography [COSMOSIL 75 SL-II-PREP, $\left.\mathrm{CHCl}_{3}-\mathrm{MeOH}(80: 1)\right]$ to afford $(S)-7 \mathbf{a}$ $(285 \mathrm{mg}, 89 \%)$ as a pale yellow amorphous. $[\alpha]_{\mathrm{D}}{ }^{26}-44.8(c$ 0.69 in $\mathrm{MeOH}) ;{ }^{1} \mathrm{H}$ NMR (400 MHz, DMSO- $\left.d_{6}\right) \delta 2.06(\mathrm{~s}$, $3 \mathrm{H}), 3.05(\mathrm{~d}, J=13.4 \mathrm{~Hz}, 1 \mathrm{H}), 3.15(\mathrm{~d}, J=13.4 \mathrm{~Hz}, 1 \mathrm{H}), 4.06$ $(\mathrm{d}, J=11.2 \mathrm{~Hz}, 1 \mathrm{H}), 4.28(\mathrm{~d}, J=11.2 \mathrm{~Hz}, 1 \mathrm{H}), 5.01-5.11(\mathrm{~m}$, 2H), 6.94-7.08 (m, 2H), 7.15-7.46 (m, 9H), 13.1 (brs, 1H); ${ }^{13} \mathrm{C}$ NMR $\left(100 \mathrm{MHz} \mathrm{CDCl}_{3}\right) \delta 20.5,30.5,36.9,63.9,64.9$, $66.6,127.0,128.0,128.2,128.3,129.6,134.1,136.0,154.4$, 170.7, 173.7; IR (neat) 3409, 3033, 2360, 1743, 1724, 1511, 1500, 1251, $1054 \mathrm{~cm}^{-1}$; ESIMS calcd for $\mathrm{C}_{20} \mathrm{H}_{21} \mathrm{NO}_{6} \mathrm{Na} \mathrm{MW}$ 394.1267, found $m / z 394.1269\left(\mathrm{M}^{+}+\mathrm{Na}\right)$.

(S)-3-Acetoxy-2-(benzyloxycarbonylamino)-2-methylpropanoic Acid [(S)-7b]

White solid, mp $85-89{ }^{\circ} \mathrm{C} ;[\alpha]_{\mathrm{D}}{ }^{27}-12.7$ (c 1.00 in $\mathrm{MeOH}) ;{ }^{1} \mathrm{H}$ NMR (400 MHz, $\left.\mathrm{CDCl}_{3}\right) \delta 1.47$ (s, 3H), 2.03 (s, $3 \mathrm{H}), 4.38$ (d, $J=11.2 \mathrm{~Hz}, 1 \mathrm{H}), 4.54$ (d, $J=11.2 \mathrm{~Hz}, 1 \mathrm{H}), 5.06$ $(\mathrm{s}, 2 \mathrm{H}), 7.26-7.40(\mathrm{~m}, 5 \mathrm{H}) ;{ }^{13} \mathrm{C} \mathrm{NMR}\left(100 \mathrm{MHz}, \mathrm{CDCl}_{3}\right) \delta$ 20.2, 20.6, 58.9, 65.7, 66.7, 127.9, 128.0, 128.3, 135.8, 154.8, 170.9, 175.1; IR (KBr) 3336, 3160, 2985, 1747, 1716, 1687, 1540, 1288, 1135, 1087, 1045, 755, $700 \mathrm{~cm}^{-1}$; ESIMS

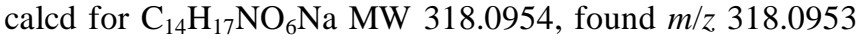
$\left(\mathrm{M}^{+}+\mathrm{Na}\right)$

\section{(S)-2-Benzyl-2-(benzyloxycarbonylamino)-3-hydroxypro- panoic Acid $[(S)-8 a]$}

To a solution of $(S)-7 \mathbf{a}(174 \mathrm{mg}, 0.468 \mathrm{mmol})$ in anhydrous THF $(7 \mathrm{~mL})$ was slowly added $1 \mathrm{M}$ solution of $\mathrm{LiEt}_{3} \mathrm{BH}(2.1 \mathrm{~mL}, 2.1 \mathrm{mmol})$ at $0{ }^{\circ} \mathrm{C}$ under argon. After being stirred at $0{ }^{\circ} \mathrm{C}$ for $30 \mathrm{~min}$, the reaction mixture was treated with $1 \mathrm{~N} \mathrm{HCl}(5 \mathrm{~mL})$ and then extracted with $\mathrm{CHCl}_{3}$ (20 mL x 6). The extract was dried over anhydrous $\mathrm{MgSO}_{4}$, filtered, and concentrated in vacuo. The oily residue was purified by silica gel column chromatography [COSMOSIL 75 SL-II-PREP, $\left.\mathrm{CHCl}_{3}-\mathrm{MeOH}(90: 1)\right]$ to afford $(S)$-8a (144 mg, 94\%, 100\% ee) as colorless needles $\left(\mathrm{CHCl}_{3}-n\right.$ hexane). After quantitative methylation of $(S)$-8a, the ee of the corresponding methyl ester was determined by HPLC analysis (CHIRALCEL OJ-H, $n$-hexane/2-propanol $=5 / 1$, $1.0 \mathrm{ml} / \mathrm{min}, 254 \mathrm{~nm}) . \mathrm{mp} 129-130{ }^{\circ} \mathrm{C} ;[\alpha]_{\mathrm{D}}{ }^{24}-66.2$ (c $1.00 \mathrm{in}$ EtOH $) ;{ }^{1} \mathrm{H}$ NMR (400 MHz, DMSO- $\left.d_{6}\right) \delta 3.06-3.18(\mathrm{~m}, 2 \mathrm{H})$, 3.37 (brs, 1H), 3.53 (d, $J=10.7 \mathrm{~Hz}, 1 \mathrm{H}), 3.62$ (d, $J=10.7 \mathrm{~Hz}$, $1 \mathrm{H}), 5.01-5.15$ (m, 2H), 6.72 (brs, 1H), 6.99-7.08 (m, 2H), 7.13-7.26 (m, 3H), 7.26-7.46 (m, 5H), 11.0-13.8 (brs, 1H); ${ }^{13} \mathrm{C}$ NMR $\left(75 \mathrm{MHz}, \mathrm{CD}_{3} \mathrm{OD}\right) \delta 37.4,64.2,67.1,67.3,127.8$, $129.06,129.10,129.14,129.5,131.1,137.1,138.4,156.6$, 174.7; IR (KBr) 3446, 3353, 3064, 3033, 2362, 2343, 1739, $1718,1685,1502,1469,1455,1394,1243,1218,1068,1049$ $\mathrm{cm}^{-1}$; EIMS calcd for $\mathrm{C}_{18} \mathrm{H}_{19} \mathrm{NO}_{5} \mathrm{MW} 329.1263$, found $\mathrm{m} / \mathrm{z}$ $329.1241\left(\mathrm{M}^{+}\right)$; Anal. Calcd for $\mathrm{C}_{18} \mathrm{H}_{19} \mathrm{NO}_{5}$ : C, 65.64; $\mathrm{H}$, 5.81 ; N, 4.25. Found: C, 65.34; H, 5.59; N, 4.20\%.

\section{(S)-2-(Benzyloxycarbonylamino)-3-hydroxy-2-methylprop- anoic Acid [(S)-8b]}

White solid, $\mathrm{mp} 123-126{ }^{\circ} \mathrm{C} ;[\alpha]_{\mathrm{D}}{ }^{29}-3.8$ (c 0.87 in $\mathrm{MeOH}) ;{ }^{1} \mathrm{H}$ NMR (400 MHz, CD $\left.3 \mathrm{OD}\right) \delta 1.46$ (s, 3H), 3.79$3.84(\mathrm{~m}, 2 \mathrm{H}), 5.06(\mathrm{~s}, 2 \mathrm{H}), 7.26-7.37(\mathrm{~m}, 5 \mathrm{H}) ;{ }^{13} \mathrm{C} \mathrm{NMR}$ $\left(100 \mathrm{MHz}, \mathrm{CD}_{3} \mathrm{OD}\right) \delta 20.5,61.6,66.0,67.2,128.5,128.7$, 129.1, 137.7, 157.0, 176.0; IR (KBr) 3440, 3291, 1733, 1691, 1556, 1459, 1267, 1174, 1089, 1047, 842, $757 \mathrm{~cm}^{-1}$;

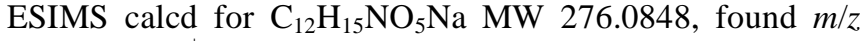
$276.0830\left(\mathrm{M}^{+}+\mathrm{Na}\right)$.

(S)-2-Amino-2-benzyl-3-hydroxypropanoic Acid [(S)- $\alpha$ Benzylserine, $(S)-9 a][11]$

The mixture of $(S)-8 \mathbf{a}(41 \mathrm{mg}, 0.124 \mathrm{mmol})$ and $10 \% \mathrm{Pd}-$ $\mathrm{C}(6.6 \mathrm{mg})$ in $\mathrm{EtOH}(5 \mathrm{~mL})$ was stirred at room temperature for $3.5 \mathrm{~h}$ under hydrogen. The reaction mixture was filtered, and concentrated in vacuo to furnish $(S)-9$ a $(22.7 \mathrm{mg}, 94 \%)$ as colorless needles $\left(\mathrm{H}_{2} \mathrm{O}-\mathrm{EtOH}\right) . \mathrm{mp} 221-222{ }^{\circ} \mathrm{C}(\mathrm{dec})$; $[\alpha]_{\mathrm{D}}^{20}+16.5\left(c 0.66\right.$ in $\left.\mathrm{H}_{2} \mathrm{O}\right)$, \{lit. $[11 \mathrm{c}]:[\alpha]_{\mathrm{D}}+16.4(c 0.81$ in $\left.\mathrm{H}_{2} \mathrm{O}\right)$, lit. [11d]: $[\alpha]_{\mathrm{D}}{ }^{20}+16.6\left(c \quad 0.9\right.$ in $\left.\mathrm{H}_{2} \mathrm{O}\right)$, lit. [11e]: $[\alpha]_{\mathrm{D}}{ }^{20}+16.0\left(c 0.71\right.$ in $\left.\left.\mathrm{H}_{2} \mathrm{O}\right)\right\} ;{ }^{1} \mathrm{H}$ NMR $\left(400 \mathrm{MHz}, \mathrm{D}_{2} \mathrm{O}\right) \delta$ $2.95(\mathrm{~d}, J=14.1 \mathrm{~Hz}, 1 \mathrm{H}), 3.26(\mathrm{~d}, J=14.1 \mathrm{~Hz}, 1 \mathrm{H}), 3.78$ (d, $J=11.9 \mathrm{~Hz}, 1 \mathrm{H}), 4.05(\mathrm{~d}, J=11.9 \mathrm{~Hz}, 1 \mathrm{H}), 7.19-7.51(\mathrm{~m}, 5 \mathrm{H})$; ${ }^{13} \mathrm{C}$ NMR $\left(75 \mathrm{MHz}, \mathrm{D}_{2} \mathrm{O}\right) \delta 40.8,67.0,69.8,130.7,131.9$, 132.8, 136.4, 176.6; IR (KBr) 3066, 2497, 2003, 1633, 1590, 1496, 1455, 1434, 1407, 1326, 1297, 1184, 1114, $1062 \mathrm{~cm}^{-1}$; ESIMS calcd for $\mathrm{C}_{10} \mathrm{H}_{14} \mathrm{NO}_{3}$ MW 196.0974, found $\mathrm{m} / \mathrm{z}$ $196.0965\left(\mathrm{M}^{+}+\mathrm{H}\right)$.

(S)-2-Amino-3-hydroxy-2-methylpropanoic Acid [(S)- $\alpha$ Methylserine, $(S)-9 b]$ [12]

Colorless needles $\left(\mathrm{H}_{2} \mathrm{O}-\mathrm{EtOH}\right) . \mathrm{mp} 222-225{ }^{\circ} \mathrm{C}(\mathrm{dec})$; $[\alpha]_{\mathrm{D}}^{28}+6.3\left(c 0.21\right.$ in $\left.\mathrm{H}_{2} \mathrm{O}\right),\left\{\right.$ lit. $[12 \mathrm{a}]:[\alpha]_{\mathrm{D}}^{22}+6.5(c 1.01$ in $\mathrm{H}_{2} \mathrm{O}$ ), lit. [12b]: $[\alpha]_{\mathrm{D}}{ }^{25}+5.3\left(c 1.02\right.$ in $\left.\left.\mathrm{H}_{2} \mathrm{O}\right)\right\} ;{ }^{1} \mathrm{H}$ NMR $(400$ $\left.\mathrm{MHz}, \mathrm{D}_{2} \mathrm{O}\right) \delta 1.43(\mathrm{~s}, 3 \mathrm{H}), 3.67(\mathrm{~d}, J=12.2 \mathrm{~Hz}, 1 \mathrm{H}), 3.92(\mathrm{~d}$, $J=12.2 \mathrm{~Hz}, 1 \mathrm{H}) ;{ }^{13} \mathrm{C} \mathrm{NMR}\left(75 \mathrm{MHz}, \mathrm{D}_{2} \mathrm{O}\right) \delta 21.3,65.3$, 67.6, 178.3; IR (KBr) 3417, 1633, 1461, 1407, 1353, 1278, $1060 \mathrm{~cm}^{-1}$; ESIMS calcd for $\mathrm{C}_{4} \mathrm{H}_{10} \mathrm{NO}_{3} \mathrm{MW} \mathrm{120.0661 \text {,found }}$ $m / z, 120.0657\left(\mathrm{M}^{+}+\mathrm{H}\right)$.

\section{(R)-2-Benzyl-2-(benzyloxycarbonylamino)-3-(methoxy- methoxy)propyl Acetate [(R)-10a]}

To a solution of $(R)-5 a(800 \mathrm{mg}, 2.238 \mathrm{mmol}, 100 \% \mathrm{ee})$ and DIEA (1.9 mL, $11.19 \mathrm{mmol})$ in anhydrous $\mathrm{CH}_{2} \mathrm{Cl}_{2}(20$ $\mathrm{mL})$ was slowly added $\mathrm{MOMCl}(850 \mu \mathrm{L}, 11.19 \mathrm{mmol})$ at 0 
${ }^{\circ} \mathrm{C}$ under argon. After being stirred at $0{ }^{\circ} \mathrm{C}$ for $30 \mathrm{~min}$, the reaction mixture was allowed to warm to room temperature and then stirred for $20.5 \mathrm{~h}$. The reaction mixture was treated with an aqueous solution saturated with $\mathrm{NH}_{4} \mathrm{Cl}(10 \mathrm{~mL})$ and then extracted with $\mathrm{CHCl}_{3}(30 \mathrm{~mL}$ x 3$)$. The extract was dried over anhydrous $\mathrm{MgSO}_{4}$, filtered, and concentrated in vacuo. The oily residue was purified by silica gel column chromatography [Silica Gel 60N, $n$-hexane-AcOEt (2:1)] to afford $(R)-\mathbf{1 0 a}(889 \mathrm{mg}, 99 \%)$ as white powder $\left(\mathrm{CHCl}_{3}-\right.$ $n$-hexane). mp $79-79.5^{\circ} \mathrm{C} ;[\alpha]_{\mathrm{D}}{ }^{24}-16.5$ (c 1.04 in $\left.\mathrm{MeOH}\right)$; ${ }^{1} \mathrm{H}$ NMR (400 MHz, $\left.\mathrm{CDCl}_{3}\right) \delta 2.05$ (s, 3H), 3.11 (d, J=13.4 $\mathrm{Hz}, 1 \mathrm{H}), 3.22$ (d, J=13.4 Hz, 1H), 3.35 (s, 3H), $3.56(\mathrm{~d}$, $J=9.5 \mathrm{~Hz}, 1 \mathrm{H}), 3.64(\mathrm{~d}, J=9.5 \mathrm{~Hz}, 1 \mathrm{H}), 4.27(\mathrm{~d}, J=11.0 \mathrm{~Hz}$, $1 \mathrm{H}), 4.31(\mathrm{~d}, J=11.0 \mathrm{~Hz}, 1 \mathrm{H}), 4.59(\mathrm{~s}, 2 \mathrm{H}), 4.95(\mathrm{~s}, 1 \mathrm{H})$, 5.06-5.15 (m, 2H), 7.06-7.13 (m, 2H), 7.16-7.28 (m, 3H), 7.28-7.46 (m, 5H); ${ }^{13} \mathrm{C} \mathrm{NMR}\left(75 \mathrm{MHz}, \mathrm{CDCl}_{3}\right) \delta 20.8,36.9$, 55.5, 57.8, 64.3, 66.4, 67.9, 96.8, 126.7, 128.1, 128.2, 128.3, $128.5,130.5,135.7,136.5,154.9,170.5$; IR (KBr) 3332, 3066, 3027, 2948, 2898, 1731, 1554, 1494, 1469, 1454, $1375,1251,1110 \mathrm{~cm}^{-1}$; ESIMS calcd for $\mathrm{C}_{22} \mathrm{H}_{27} \mathrm{NO}_{6} \mathrm{Na} \mathrm{MW}$ 424.1736, found $m / z 424.1736\left(\mathrm{M}^{+}+\mathrm{Na}\right)$; Anal. Calcd for $\mathrm{C}_{22} \mathrm{H}_{27} \mathrm{NO}_{6}$ : C, 65.82; H, 6.78; N, 3.49. Found: C, 65.64; H, $6.71 ; \mathrm{N}, 3.50 \%$.

\section{(R)-2-(Benzyloxycarbonylamino)-3-(methoxymethoxy)-2- methylpropyl Acetate [(R)-10b]}

Colorless oil; $[\alpha]_{\mathrm{D}}{ }^{29}-1.8$ (c 1.14 in $\left.\mathrm{MeOH}\right) ;{ }^{1} \mathrm{H}$ NMR $\left(400 \mathrm{MHz}, \mathrm{CDCl}_{3}\right) \delta 1.40(\mathrm{~s}, 3 \mathrm{H}), 2.07(\mathrm{~s}, 3 \mathrm{H}), 3.33(\mathrm{~s}, 3 \mathrm{H})$, $3.54(\mathrm{~d}, J=9.8 \mathrm{~Hz}, 1 \mathrm{H}), 3.65(\mathrm{~d}, J=9.8 \mathrm{~Hz}, 1 \mathrm{H}), 4.20$ (d, $J=11.2 \mathrm{~Hz}, 1 \mathrm{H}), 4.31$ (d, $J=11.0 \mathrm{~Hz}, 1 \mathrm{H}), 4.60$ (s, 2H), 5.06 $(\mathrm{s}, 2 \mathrm{H}), 5.18(\mathrm{~s}, 1 \mathrm{H}), 7.30-7.35(\mathrm{~m}, 5 \mathrm{H}) ;{ }^{13} \mathrm{C}$ NMR $(100$ $\left.\mathrm{MHz} \mathrm{CDCl}_{3}\right) \delta 19.2,20.8,54.9,55.3,65.9,66.3,70.3,96.6$, $127.9,128.3,136.3,154.7,170.5$; IR (neat) 3351, 2946, 1733, 1523, 1455, 1376, 1238, 1147, 1047, 917, 742, 698

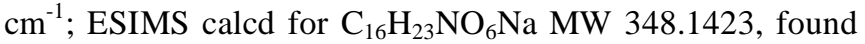
$m / z, 348.1427\left(\mathrm{M}^{+}+\mathrm{Na}\right)$.

\section{Benzyl (S)-2-Benzyl-1-hydroxy-3-(methoxymethoxy)pro-} pan-2-ylcarbamate $[(S)-11 a]$

To a solution of $(R)-\mathbf{1 0 a}(159 \mathrm{mg}, 0.396 \mathrm{mmol})$ in anhydrous THF $(10 \mathrm{~mL})$ was slowly added $1 \mathrm{M}$ solution of $\mathrm{LiEt}_{3} \mathrm{BH}(1.18 \mathrm{~mL}, 1.18 \mathrm{mmol})$ at $0{ }^{\circ} \mathrm{C}$ under argon. After being stirred at $0{ }^{\circ} \mathrm{C}$ for $50 \mathrm{~min}$, the reaction mixture was treated with $1 \mathrm{~N} \mathrm{HCl}(5 \mathrm{~mL})$ and then extracted with $\mathrm{CHCl}_{3}$ (20 mL x 3). The extract was dried over anhydrous $\mathrm{MgSO}_{4}$, filtered, and concentrated in vacuo. The oily residue was purified by silica gel column chromatography [Silica Gel $60 \mathrm{~N}, n$-hexane-AcOEt $(1: 1)]$ to afford $(S)$-11a (127 mg, $89 \%)$ as a colorless oil. $[\alpha]_{\mathrm{D}}{ }^{28}+8.1\left(c 0.94\right.$ in $\left.\mathrm{CHCl}_{3}\right) ;{ }^{1} \mathrm{H}$ NMR (400 MHz, $\left.\mathrm{CDCl}_{3}\right) \delta 2.91(\mathrm{~d}, J=13.4 \mathrm{~Hz}, 1 \mathrm{H}), 3.17(\mathrm{~d}$, $J=13.4 \mathrm{~Hz}, 1 \mathrm{H}), 3.36$ (s, 3H), 3.45 (d, J=9.7 Hz, 1H), 3.63 $(\mathrm{d}, J=9.7 \mathrm{~Hz}, 1 \mathrm{H}), 3.66-3.80(\mathrm{~m}, 3 \mathrm{H}), 4.62(\mathrm{~s}, 2 \mathrm{H}), 5.11(\mathrm{~s}$, 2H), $5.22(\mathrm{~s}, 1 \mathrm{H}), 7.11-7.29(\mathrm{~m}, 5 \mathrm{H}), 7.29-7.41(\mathrm{~m}, 5 \mathrm{H}) ;{ }^{13} \mathrm{C}$ NMR $\left(100 \mathrm{MHz}, \mathrm{CDCl}_{3}\right) \delta 37.3,55.5,59.3,65.1,66.5,69.4$, $96.8,126.4,127.9,128.0,128.1,128.3,130.3,135.8,136.2$, 155.8; IR (neat) 3413, 3336, 3062, 3029, 2946, 2886, 1716, 1513, 1454, 1251, $1220 \mathrm{~cm}^{-1}$; ESIMS calcd for $\mathrm{C}_{20} \mathrm{H}_{25} \mathrm{NO}_{5} \mathrm{Na} \mathrm{MW} 382.1630$, found $\mathrm{m} / \mathrm{z} 382.1630\left(\mathrm{M}^{+}+\right.$ $\mathrm{Na}$.
Benzyl (S)-1-Hydroxy-3-(methoxymethoxy)-2-methylpropan-2-ylcarbamate [(S)-11b]

Colorless oil; $[\alpha]_{\mathrm{D}}{ }^{28}-1.7$ (c 0.96 in $\left.\mathrm{MeOH}\right) ;{ }^{1} \mathrm{H}$ NMR $\left(400 \mathrm{MHz}, \mathrm{CDCl}_{3}\right) \delta 1.29(\mathrm{~s}, 3 \mathrm{H}), 3.34(\mathrm{~s}, 3 \mathrm{H}), 3.51-3.77$ $(\mathrm{m}, 5 \mathrm{H}), 4.61(\mathrm{~s}, 2 \mathrm{H}), 5.08(\mathrm{~s}, 2 \mathrm{H}), 5.40(\mathrm{~s}, 1 \mathrm{H}), 7.31-7.36$ $(\mathrm{m}, 5 \mathrm{H}) ;{ }^{13} \mathrm{C}$ NMR $\left(100 \mathrm{MHz}, \mathrm{CDCl}_{3}\right) \delta 19.6,55.3,56.4$, $66.5,67.5,71.6,96.6,127.9,128.0,128.3,136.2,155.8$; IR (neat) $3415,2940,1706,1511,1454,1245,1147,1045,742$, $698 \mathrm{~cm}^{-1}$; ESIMS calcd for $\mathrm{C}_{14} \mathrm{H}_{21} \mathrm{NO}_{5} \mathrm{Na} \mathrm{MW} 306.1317$, found $m / z 306.1317\left(\mathrm{M}^{+}+\mathrm{Na}\right)$.

\section{Benzyl (R)-2-Benzyl-1-(methoxymethoxy)-3-oxopropan-} 2-ylcarbamate $[(R)-12 a]$

To a solution of $(S)$-11a $(470 \mathrm{mg}, 1.307 \mathrm{mmol})$ and TEA (1.82 $\mathrm{mL}, 13.07 \mathrm{mmol})$ in anhydrous DMSO $(10 \mathrm{~mL})$ was added $\mathrm{SO}_{3}$-pyridine $(1.66 \mathrm{~g}, 10.46 \mathrm{mmol})$ at room temperature under argon. After being stirred at room temperature for $40 \mathrm{~min}$, the reaction mixture was treated with $1 \mathrm{~N} \mathrm{HCl} \mathrm{(5}$ $\mathrm{mL})$ and then extracted with $\mathrm{CHCl}_{3}(20 \mathrm{~mL} \times 3)$. The extract was dried over anhydrous $\mathrm{MgSO}_{4}$, filtered, and concentrated in vacuo. The oily residue was purified by silica gel column chromatography [Silica Gel $60 \mathrm{~N}, n$-hexane-AcOEt (2:1)] to afford $(R)-\mathbf{1 2 a}(418 \mathrm{mg}, 90 \%)$ as colorless needles $\left(\mathrm{CHCl}_{3}-n\right.$-hexane). mp $72-72.5^{\circ} \mathrm{C} ;[\alpha]_{\mathrm{D}}{ }^{24}-2.1$ (c 1.00 in $\mathrm{MeOH}) ;{ }^{1} \mathrm{H}$ NMR (400 MHz, $\left.\mathrm{CDCl}_{3}\right) \delta 3.15-3.36(\mathrm{~m}, 5 \mathrm{H})$, 3.86-4.02 (m, 2H), $4.55(\mathrm{~s}, 2 \mathrm{H}), 5.09(\mathrm{~d}, J=12.2 \mathrm{~Hz}, 1 \mathrm{H})$, $5.17(\mathrm{~d}, J=12.2 \mathrm{~Hz}, 1 \mathrm{H}), 5.50(\mathrm{~s}, 1 \mathrm{H}), 6.93-7.00(\mathrm{~m}, 2 \mathrm{H})$, 7.14-7.28 (m, 3H), 7.28-7.42 (m, 5H), $9.59(\mathrm{~s}, 1 \mathrm{H}) ;{ }^{13} \mathrm{C}$ NMR $\left(75 \mathrm{MHz}, \mathrm{CDCl}_{3}\right) \delta 35.6,55.5,66.6,66.7,67.8,96.7$, $127.0,128.20,128.23,128.4,128.5,130.0,134.3,136.3$, 155.0, 199.1; IR (KBr) 3357, 3066, 2942, 1716, 1508, 1255, $1037 \mathrm{~cm}^{-1}$; ESIMS calcd for $\mathrm{C}_{20} \mathrm{H}_{23} \mathrm{NO}_{5} \mathrm{Na} \mathrm{MW} 380.1474$, found $m / z 380.1507\left(\mathrm{M}^{+}+\mathrm{Na}\right)$; Anal. Calcd for $\mathrm{C}_{20} \mathrm{H}_{23} \mathrm{NO}_{5}$ : C, 67.21; H, 6.49; N, 3.92. Found: C, 67.06; H, 6.56; N, $3.90 \%$.

Benzyl (R)-1-(Methoxymethoxy)-2-methyl-3-oxopropan-2ylcarbamate [(R)-12b]

Colorless oil; $[\alpha]_{\mathrm{D}}{ }^{27}+18.3$ (c 0.77 in $\left.\mathrm{MeOH}\right) ;{ }^{1} \mathrm{H}$ NMR $\left(400 \mathrm{MHz}, \mathrm{CDCl}_{3}\right) \delta 1.44(\mathrm{~s}, 3 \mathrm{H}), 3.29(\mathrm{~s}, 3 \mathrm{H}), 3.75-3.85$ $(\mathrm{m}, 2 \mathrm{H}), 4.57$ (s, 2H), $5.11(\mathrm{~s}, 2 \mathrm{H}), 5.70(\mathrm{~s}, 1 \mathrm{H}), 7.30-7.37$ $(\mathrm{m}, 5 \mathrm{H}), 9.50(\mathrm{~s}, 1 \mathrm{H}) ;{ }^{13} \mathrm{C} \mathrm{NMR}\left(100 \mathrm{MHz}, \mathrm{CDCl}_{3}\right) \delta 17.2$, $55.3,62.7,66.7,68.9,96.5,127.9,128.0,128.3,136.0$, 155.1, 199.4; IR (neat) 3342, 2946, 1714, 1517, 1455, 1402, 1259, 1149, 1045, 777, 742, $698 \mathrm{~cm}^{-1}$; ESIMS calcd for $\mathrm{C}_{14} \mathrm{H}_{19} \mathrm{NO}_{5} \mathrm{Na} \mathrm{MW} 304.1161$, found $\mathrm{m} / \mathrm{z} 304.1164\left(\mathrm{M}^{+}+\right.$ $\mathrm{Na})$.

\section{(R)-2-Benzyl-2-(benzyloxycarbonylamino)-3-(methoxy-} methoxy)propanoic Acid [( $R)-13 a]$.

To a solution of $(R)-\mathbf{1 2 a}(353 \mathrm{mg}, 0.987 \mathrm{mmol})$, 2methyl-2-butene $(418 \mu \mathrm{L}, 3.95 \mathrm{mmol})$ and $\mathrm{NaH}_{2} \mathrm{PO}_{4}(154$ $\mathrm{mg}, 0.987 \mathrm{mmol})$ in $\mathrm{H}_{2} \mathrm{O}$-tert-BuOH $(1: 5,12 \mathrm{~mL})$ was added $\mathrm{NaClO}_{2}(268 \mathrm{mg}, 2.96 \mathrm{mmol})$ at room temperature. After being stirred at room temperature for $1 \mathrm{~h}$, the reaction mixture was treated with $1 \mathrm{~N} \mathrm{HCl}(5 \mathrm{~mL})$ and then extracted with $\mathrm{CHCl}_{3}(20 \mathrm{~mL}$ x 6$)$. The extract was dried over anhydrous $\mathrm{MgSO}_{4}$, filtered, and concentrated in vacuo. An aqueous solution saturated with $\mathrm{NaHCO}_{3}(5 \mathrm{~mL})$ was added to the concentrated solution and then the aqueous layer was 
washed with $\mathrm{CHCl}_{3}(5 \mathrm{~mL})$. The aqueous layer was acidified with $1 \mathrm{~N} \mathrm{HCl}(10 \mathrm{~mL})$ and then extracted with $\mathrm{CHCl}_{3}(20$ $\mathrm{mL}$ x 6). The extract was dried over anhydrous $\mathrm{MgSO}_{4}$, filtered, and concentrated in vacuo to gave $(R)-\mathbf{1 3 a}(336 \mathrm{mg}$, $91 \%)$ as a white powder. $\mathrm{mp} 59-60{ }^{\circ} \mathrm{C} ;[\alpha]_{\mathrm{D}}{ }^{17}+55.4(c 0.66$ in $\left.\mathrm{CHCl}_{3}\right) ;{ }^{1} \mathrm{H}$ NMR (400 $\left.\mathrm{MHz}, \mathrm{CDCl}_{3}\right) \delta 3.13(\mathrm{~d}, J=13.4$ $\mathrm{Hz}, 1 \mathrm{H}), 3.28(\mathrm{~s}, 3 \mathrm{H}), 3.50(\mathrm{~d}, J=13.4 \mathrm{~Hz}, 1 \mathrm{H}), 3.95(\mathrm{~d}$, $J=9.3 \mathrm{~Hz}, 1 \mathrm{H}), 4.26(\mathrm{~d}, J=9.3 \mathrm{~Hz}, 1 \mathrm{H}), 4.59$ (s, 2H), 5.07 (d, $J=12.2 \mathrm{~Hz}, 1 \mathrm{H}), 5.20(\mathrm{~d}, J=12.2 \mathrm{~Hz}, 1 \mathrm{H}), 5.66$ (s, 1H), 6.96$7.07(\mathrm{~m}, 2 \mathrm{H}), 7.12-7.28(\mathrm{~m}, 3 \mathrm{H}), 7.28-7.43(\mathrm{~m}, 5 \mathrm{H}) ;{ }^{13} \mathrm{C}$ NMR $\left(75 \mathrm{MHz}, \mathrm{CDCl}_{3}\right) \delta 36.9,55.3,65.0,66.5,68.9,96.5$, 127.1, 128.1, 128.3, 128.4, 129.8, 134.7, 136.4, 154.7, 176.2; IR (KBr) 3374, 3033, 2950, 1739, 1716, 1509, 1455, 1405, 1257, 1214, 1151, 1112, $1056 \mathrm{~cm}^{-1}$; ESIMS calcd for $\mathrm{C}_{20} \mathrm{H}_{23} \mathrm{NO}_{6} \mathrm{Na}$ MW 396.1423, found $\mathrm{m} / \mathrm{z} 396.1429\left(\mathrm{M}^{+}+\right.$ $\mathrm{Na}$; Anal. Calcd for $\mathrm{C}_{20} \mathrm{H}_{23} \mathrm{NO}_{6}: \mathrm{C}, 64.33 ; \mathrm{H}, 6.21 ; \mathrm{N}, 3.75$. Found: C, 64.03; H, 6.19; N, 3.68\%.

(R)-2-(Benzyloxycarbonylamino)-3-(methoxymethoxy)-2propanoic Acid [(R)-13b]

Colorless oil; $[\alpha]_{\mathrm{D}}{ }^{27}+1.3$ (c 0.98 in $\left.\mathrm{MeOH}\right) ;{ }^{1} \mathrm{H}$ NMR (400 MHz, CD 3 OD) $\delta 1.53(\mathrm{~s}, 3 \mathrm{H}), 3.26(\mathrm{~s}, 3 \mathrm{H}), 3.82(\mathrm{~d}$, $J=9.8 \mathrm{~Hz}, 1 \mathrm{H}), 3.88(\mathrm{~d}, J=9.8 \mathrm{~Hz}, 1 \mathrm{H}), 4.56(\mathrm{~s}, 2 \mathrm{H}), 5.06(\mathrm{~s}$, $2 \mathrm{H}), 7.25-7.40(\mathrm{~m}, 5 \mathrm{H}) ;{ }^{13} \mathrm{C} \mathrm{NMR}\left(75 \mathrm{MHz}, \mathrm{CDCl}_{3}\right) \delta 20.1$, $55.4,60.0,66.7,70.6,96.6,128.1,128.2,128.5,136.2$, 155.2, 177.1; IR (neat) 2948, 1714, 1506, 1454, 1257, 1043, 977, 917, 754, $698 \mathrm{~cm}^{-1}$; ESIMS calcd for $\mathrm{C}_{14} \mathrm{H}_{19} \mathrm{NO}_{6} \mathrm{Na}$ MW 320.1110, found $\mathrm{m} / \mathrm{z} 320.1108\left(\mathrm{M}^{+}+\mathrm{Na}\right)$.

(R)-2-Benzyl-2-(benzyloxycarbonylamino)-3-hydroxypropanoic Acid [(R)-8a]

To a solution of $(R)-\mathbf{1 3 a}(280 \mathrm{mg}, 0.75 \mathrm{mmol})$ in anhydrous THF $(10 \mathrm{~mL})$ was added $1 \mathrm{~N} \mathrm{HCl}(5 \mathrm{~mL})$ at room temperature. After being refluxed for $24 \mathrm{~h}$, the reaction mixture was extracted with $\mathrm{CHCl}_{3}(20 \mathrm{~mL}$ x 8$)$. The extract was dried over anhydrous $\mathrm{MgSO}_{4}$, filtered, and concentrated in vacuo. The oily residue was purified by silica gel column chromatography [COSMOSIL 75 SL-II-PREP, $\mathrm{CHCl}_{3}-$ $\mathrm{MeOH}(90: 1)]$ to afford $(R)-8 \mathbf{a}(231 \mathrm{mg}, 94 \%, 100 \%$ ee) as colorless needles $\left(\mathrm{CHCl}_{3}-n\right.$-hexane $) . \operatorname{mp} 129-130{ }^{\circ} \mathrm{C}$; $[\alpha]_{\mathrm{D}}{ }^{24}+65.8\left(c 1.00\right.$ in EtOH); Anal. Calcd for $\mathrm{C}_{18} \mathrm{H}_{19} \mathrm{NO}_{5}$ : C, 65.64; H, 5.81; N, 4.25. Found: C, 65.35; H, 5.89; N, $4.25 \%$.

\section{(R)-2-(Benzyloxycarbonylamino)-3-hydroxy-2-methylpro- panoic Acid [(R)-8b]}

White solid, mp 124-127 ${ }^{\circ} \mathrm{C} ;[\alpha]_{\mathrm{D}}{ }^{27}+3.7$ (c 1.04 in $\mathrm{MeOH})$; ESIMS calcd for $\mathrm{C}_{12} \mathrm{H}_{15} \mathrm{NO}_{5} \mathrm{Na} \mathrm{MW} 276.0848$, found $\mathrm{m} / \mathrm{z} 276.0843\left(\mathrm{M}^{+}+\mathrm{Na}\right)$.

\section{(R)-2-Amino-2-benzyl-3-hydroxypropanoic Acid $[(R)-\alpha$ - Benzylserine, $(R)-9 a] ~[11]$}

The mixture of $(R)-8 \mathbf{a}(256 \mathrm{mg}, 0.777 \mathrm{mmol})$ and $10 \%$ $\mathrm{Pd}-\mathrm{C}(41 \mathrm{mg})$ in EtOH $(15 \mathrm{~mL})$ was stirred at room temperature for $4 \mathrm{~h}$ under hydrogen. The reaction mixture was submitted to filtration with celite, and concentrated in vacuo to furnish $(R)-9$ a $(152 \mathrm{mg}, 100 \%)$ as colorless needles $\left(\mathrm{H}_{2} \mathrm{O}-\right.$ EtOH). mp 221-222 ${ }^{\circ} \mathrm{C}(\mathrm{dec}) ;[\alpha]_{\mathrm{D}}{ }^{24}-16.1\left(c 0.69\right.$ in $\left.\mathrm{H}_{2} \mathrm{O}\right)$; ESIMS calcd for $\mathrm{C}_{10} \mathrm{H}_{14} \mathrm{NO}_{3}$ MW 196.0974, found $\mathrm{m} / \mathrm{z}$ $196.0963\left(\mathrm{M}^{+}+\mathrm{H}\right)$.
(R)-2-Amino-3-hydroxy-2-methylpropanoic Acid [(R)- $\alpha$ Methylserine, (R)-9b] [12]

Colorless needles $\left(\mathrm{H}_{2} \mathrm{O}-\mathrm{EtOH}\right) . \mathrm{mp} 260-265{ }^{\circ} \mathrm{C}$ (dec); $[\alpha]_{\mathrm{D}}{ }^{29}-5.7\left(c 0.22\right.$ in $\left.\mathrm{H}_{2} \mathrm{O}\right)$; ESIMS calcd for $\mathrm{C}_{4} \mathrm{H}_{10} \mathrm{NO}_{3} \mathrm{MW}$ 120.0661 , found $m / z 120.0655\left(\mathrm{M}^{+}+\mathrm{H}\right)$.

Enzyme-catalyzed Hydrolysis of Diethyl 2(Benzyloxycarbonylamino)-2-methylmalonate (3b) with Porcine Liver Esterase

PLE (Sigma; E-2884, $1.6 \mathrm{~mL}, 6,000$ units) was added to a stirred solution of $3 \mathbf{b}(1.5 \mathrm{~g}, 4.64 \mathrm{mmol})$ in $1 / 15 \mathrm{M}$ phosphate buffer $(\mathrm{pH} 7.0,405 \mathrm{~mL})$ and $\mathrm{MeCN}(46 \mathrm{~mL})$ at room temperature. After being stirred at room temperature for $6 \mathrm{~h}$, the reaction mixture was treated with $1 \mathrm{~N} \mathrm{HCl}(30 \mathrm{~mL})$ and then extracted with AcOEt (200 mL x 5). The extract was dried over anhydrous $\mathrm{MgSO}_{4}$, filtered, and concentrated in vacuo. The oily residue was purified by silica gel column chromatography [COSMOSIL 75 SL-II-PREP, $\mathrm{CHCl}_{3}-$ $\mathrm{MeOH}(9: 1)]$ to afford $(R)-14(1.34 \mathrm{~g}, 98 \%, 97 \%$ ee) as a colorless amorphous. $[\alpha]_{\mathrm{D}}{ }^{28}+25.8(c 0.45$ in $\mathrm{MeOH}) ;{ }^{1} \mathrm{H}$ NMR (400 MHz, DMSO- $\left.d_{6}\right) \delta$ 1.02-1.22 (m, 3H), $1.57(\mathrm{~s}$, $3 \mathrm{H}), 3.98-4.20(\mathrm{~m}, 2 \mathrm{H}), 5.03(\mathrm{~d}, J=12.9 \mathrm{~Hz}, 1 \mathrm{H}), 5.05$ (d, $J=12.7 \mathrm{~Hz}, 1 \mathrm{H}), 7.23-7.57$ (m, 6H), 13.4 (brs, $1 \mathrm{H}) ;{ }^{13} \mathrm{C} \mathrm{NMR}$ $\left(100 \mathrm{MHz}, \mathrm{DMSO}-d_{6}\right) \delta 13.8,21.5,61.4,62.9,65.6,127.5$, $127.8,128.2,136.7,154.5,168.5,169.8$; IR (neat) 2985, 2360, 1732, 1506, 1456, 1275, 1119, 1066, $1020 \mathrm{~cm}^{-1}$;

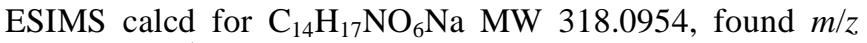
$318.0932\left(\mathrm{M}^{+}+\mathrm{Na}\right)$.

(S)-2-(Benzyloxycarbonylamino)-3-hydroxy-2-methylpropanoic Acid $[(S)-8 b]$

To a solution of $(R)-\mathbf{1 4}(130 \mathrm{mg}, 0.440 \mathrm{mmol})$ in THF (7 $\mathrm{mL})$ was slowly added $1 \mathrm{M}$ solution of $\mathrm{LiEt}_{3} \mathrm{BH}(2.2 \mathrm{~mL}, 2.2$ mmol) at $0{ }^{\circ} \mathrm{C}$ under argon. After being stirred at $0{ }^{\circ} \mathrm{C}$ for 40 $\min$, the reaction mixture was treated with $1 \mathrm{~N} \mathrm{HCl}(10 \mathrm{~mL})$ and then extracted with $\mathrm{CHCl}_{3}(20 \mathrm{~mL}$ x 6). The extract was dried over anhydrous $\mathrm{MgSO}_{4}$, filtered, and concentrated in vасио. The oily residue was purified by silica gel column chromatography [COSMOSIL 75 SL-II-PREP, $\mathrm{CHCl}_{3}-$ $\mathrm{MeOH}(80: 1)]$ to afford $(S)-8 \mathbf{b}(88 \mathrm{mg}, 79 \%)$ as a pale yellow amorphous. $[\alpha]_{\mathrm{D}}^{22}-3.6(c 0.65$ in $\mathrm{MeOH})$.

Ethyl (S)-2-(Benzyloxycarbonylamino)-3-hydroxy-2methylpropanoate $[(S)-15]$

To a solution of $(S)-8 \mathbf{b}(77 \mathrm{mg}, 0.304 \mathrm{mmol})$ in acetone ( $7 \mathrm{~mL}$ ) was added EtI $(244 \mu \mathrm{L}, 3.04 \mathrm{mmol})$ and $\mathrm{K}_{2} \mathrm{CO}_{3}(46$ $\mathrm{mg}, 0.344 \mathrm{mmol})$ at room temperature. After being refluxed for $2 \mathrm{~h}$, the reaction mixture was treated with $1 \mathrm{~N} \mathrm{HCl} \mathrm{(5}$ $\mathrm{mL})$ and then extracted with $\mathrm{CHCl}_{3}(15 \mathrm{~mL}$ x 3$)$. The extract was dried over anhydrous $\mathrm{MgSO}_{4}$, filtered, and concentrated in vacuo. The oily residue was purified by silica gel column chromatography [Silica Gel $60 \mathrm{~N}, n$-hexane-AcOEt (2:1)] to afford $(S)-\mathbf{1 5}(71 \mathrm{mg}, 84 \%, 96 \%$ ee) as a colorless oil. The ee of $(S)$-15 was determined by HPLC analysis (CHIRALPAK AD-H, $n$-hexane/2-propanol = 10/1, $1.0 \mathrm{ml} / \mathrm{min}, 254$ $\mathrm{nm}) .[\alpha]_{\mathrm{D}}{ }^{28}+3.2$ (c 3.04 in $\left.\mathrm{CHCl}_{3}\right) ;{ }^{1} \mathrm{H} \mathrm{NMR}(400 \mathrm{MHz}$, $\left.\mathrm{CDCl}_{3}\right) \delta 1,26(\mathrm{t}, J=6.8 \mathrm{~Hz}, 3 \mathrm{H}) ; 1.51(\mathrm{~s}, 3 \mathrm{H}), 2.81-2.94(\mathrm{~m}$, $1 \mathrm{H}), 3.81(\mathrm{dd}, J=7.8,11.0 \mathrm{~Hz}, 1 \mathrm{H}) 4.05(\mathrm{dd}, J=5.6,11.0 \mathrm{~Hz}$, $1 \mathrm{H}), 4.15-4.30(\mathrm{~m}, 2 \mathrm{H}), 5.09(\mathrm{~s}, 2 \mathrm{H}), 5.67(\mathrm{~s}, 1 \mathrm{H}), 7.28-7.40$ $(\mathrm{m}, 5 \mathrm{H}) ;{ }^{13} \mathrm{C} \mathrm{NMR}\left(100 \mathrm{MHz}, \mathrm{CDCl}_{3}\right) \delta 14.0,20.3,61.2$, $61.8,66.3,66.7,127.9,128.0,128.3,135.9,155.3,172.8$; IR 
(neat) 3361, 2983, 1716, 1508, 1455, 1373, $1249 \mathrm{~cm}^{-1}$; ESIMS calcd for $\mathrm{C}_{14} \mathrm{H}_{19} \mathrm{NO}_{5} \mathrm{Na}$ MW 304.1161, found $\mathrm{m} / \mathrm{z}$ $304.1147\left(\mathrm{M}^{+}+\mathrm{Na}\right)$.

\section{Ethyl (R)-2-(Benzyloxycarbonylamino)-3-hydroxy-2- methylpropanoate $[(R)-15]$}

To a solution of $(R)-\mathbf{1 4}(100 \mathrm{mg}, 0.34 \mathrm{mmol})$ in anhydrous THF $(5 \mathrm{~mL})$ was added DAST $(56.8 \mu \mathrm{L}, 0.41 \mathrm{mmol})$ at $0{ }^{\circ} \mathrm{C}$ under argon. After being stirred for $2 \mathrm{~h}$ at room temperature, the reaction mixture was concentrated in vacuo, treated with ice water $(3 \mathrm{~mL})$ and then extracted with $\mathrm{CH}_{2} \mathrm{Cl}_{2}$ $(10 \mathrm{~mL} \times 3)$. The organic layer was dried over anhydrous $\mathrm{MgSO}_{4}$, filtered, and concentrated in vacuo. To the concentrated solution (ca. $2 \mathrm{~mL}$ ) was added $\mathrm{NaBH}_{4}(19.2 \mathrm{mg}, 0.51$ mmol) at $-78{ }^{\circ} \mathrm{C}$ under argon, and then $\mathrm{MeOH}(5 \mathrm{~mL})$ was slowly added to the solution at $-78{ }^{\circ} \mathrm{C}$ over $3 \mathrm{~min}$. After being stirred at $-78{ }^{\circ} \mathrm{C}$ for $1 \mathrm{~h}$, the reaction mixture was treated with $1 \mathrm{~N} \mathrm{HCl}(5 \mathrm{~mL})$ and then extracted with $\mathrm{CHCl}_{3}(15 \mathrm{~mL}$ x 3). The extract was dried over anhydrous $\mathrm{MgSO}_{4}$, filtered, and concentrated in vacuo. The oily residue was purified by silica gel column chromatography [Silica Gel $60 \mathrm{~N}, n-$ hexane-AcOEt (1:1)] to afford $(R)-\mathbf{1 5}(79 \mathrm{mg}, 83 \%, 97 \%$ ee) as a colorless oil. The ee of $(R)-\mathbf{1 5}$ was determined by HPLC analysis (CHIRALPAK AD-H, $n$-hexane/2-propanol $=10 / 1,1.0 \mathrm{ml} / \mathrm{min}, 254 \mathrm{~nm}$ ). $[\alpha]_{\mathrm{D}}{ }^{28}-3.0$ (c 3.14 in $\left.\mathrm{CHCl}_{3}\right)$; ESIMS calcd for $\mathrm{C}_{14} \mathrm{H}_{19} \mathrm{NO}_{5} \mathrm{Na}$ MW 304.1161, found $\mathrm{m} / \mathrm{z}$ $304.1185\left(\mathrm{M}^{+}+\mathrm{Na}\right)$.

\section{ACKNOWLEDGEMENTS}

This work was supported in part by a Grant-in-Aid for Scientific Research (C) from the Japan Society for the Promotion of Science. The authors also greatly appreciate a research grant from the Research Foundation for Pharmaceutical Sciences.

\section{REFERENCES AND NOTES}

[1] (a) Wirth, T. In Organic Synthesis Highlights IV; Schmalz, H.-G., Ed.; Wiley-VCH: Weinheim, 2000; pp 26-33. (b) Sano, S.; Nagao, Y. J. Synth. Org. Chem., Jpn., 2000, 58, 756. (c) Matsushita, M.; Maeda, H.; Kodama, M. Recent Res. Develop. Org. Chem., 2002, 6, 587. (d) Sato, H.; Oishi, T.; Chida, N. J. Synth. Org. Chem., Jpn., 2004, 62, 693. (e) Ofune, Y.; Shinada, T. Eur. J. Org. Chem., 2005, 5127. (f) Maruoka, K. J. Synth. Org. Chem. Jpn., 2005, 63, 686. (g) Byun, H.-S.; Lu, X.; Bittman, R. Synthesis, 2006, 2447; Vogt, H.; Bräse, S. Org. Biomol. Chem., 2007, 5, 406.

[2] Just, G.; Payette, D.R. Tetrahedron Lett., 1980, 21, 3219. (b) Banfi, L.; Beretta, M. G.; Colombo, L.; Gennari, C.; Scolastico, C. J. Chem. Soc., Perkin Trans. 1, 1983, 1613. (c) Yoshikawa, M.; Yokokama, Y.; Okuno, Y.; Yagi, N.; Murakami, N. Chem. Pharm. Bull., 1994, 42, 2662. (d) Yoshikawa, M.; Yokokama, Y.; Okuno, Y.; Murakami, N. Tetrahedron, 1995, 51, 6209. (e) Sano, S.; Kobayashi, Y.; Kondo, T.; Takebayashi, M.; Maruyama, S.; Fujita, T.; Nagao, Y. Tetrahedron Lett., 1995, 36, 2097. (f) Hatakeyama, S.; Yoshida, M.; Esumi, T.; Iwabuchi, Y.; Irie, H.; Kawamoto, T.; Yamada, H.; Nishizawa, M. Tetrahedron Lett., 1997, 38, 7887. (g) Rao, A.V.R.; Gurjar, M.K.; Devi, T.R.; Kumar, K.R. Tetrahedron Lett., 1993, 34, 1653. (h) Deloisy, S.; Thang, T.T.; Olesker, A.; Lukacs, G. Tetrahedron Lett., 1994, 35, 4783. (i) Oishi, T.; Ando, K.; Inomiya, K.; Sato, H.; Iida, M.; Chida, N. Bull. Chem. Soc., Jpn., 2002, 75, 1927. (j) Gonda, J.; Martinková, M.; Raschmanová, J.; Balentová, E. Tetrahedron Lett., 2006, 17, 1875.

[3] Kobayashi, S.; Hayashi, T.; Iwamoto, S.; Furuta, T.; Matsumura, M. Synlett, 1996, 672. (b) Kobayashi, S.; Matsumura, M.; Furuta, T.; Hayashi, T.; Iwamoto, S. Synlett, 1997, 301. (c) Kobayashi, S.; Furuta, T.; Hayashi, T.; Nishijima, M.; Hanada, K. J. Am. Chem.
Soc., 1998, 120, 908. (d) Kobayashi, S.; Furuta, T. Tetrahedron, 1998, 54, 10275. (e) Wang, B.; Yu, X.; Lin, G. Synlett, 2001, 904. (f) Oishi, T.; Ando, K.; Inomiya, K.; Sato, H.; Iida, M.; Chida, N. Org. Lett., 2002, 4, 151.

[4] Hatakeyama, S.; Fukuyama, H.; Mukugi, Y.; Irie, H. Tetrahedron Lett., 1996, 37, 4047. (b) Sano, S.; Miwa, T.; Hayashi, K.; Nozaki, K.; Ozaki, Y.; Nagao, Y. Tetrahedron Lett., 2001, 42, 4029. (c) Matsukawa, Y.; Isobe, M.; Kotsuki, H.; Ichikawa, Y. J. Org. Chem., 2005, 70, 5339. (d) Chakraborty, T.K.; Sudhakar, G. Tetrahedron Lett., 2006, 47, 5847. (e) Yakura, T.; Yoshimoto, Y.; Ishikawa, C.; Mabuchi, S. Tetrahedron, 2007, 63, 4429.

[5] Uno, H.; Baldwin, J.E.; Russell, A.T. J. Am. Chem. Soc., 1994, 116, 2139. (b) Chida, N.; Takeoka, J.; Tsutsumi, N.; Ogawa, S. J. Chem. Soc., Chem. Commun., 1995, 793. (c) Nagamitsu, T.; Sunazuka, T.; Tanaka, H.; Omura, S.; Sprengeler, P.A.; Smith, A.B., III J. Am. Chem. Soc., 1996, 118, 3584. (d) Corey, E.J.; Li, W.; Nagamitsu, T. Angew. Chem. Int. Ed., 1998, 37, 1676. (e) Panek, J.S.; Masse, C.E. Angew. Chem. Int. Ed., 1999, 38, 1093. (f) Ooi, H.; Ishibashi, N.; Iwabuchi, Y.; Ishihara, J.; Hatakeyama, S. J. Org. Chem., 2004, 69, 7765. (g) Fukuda, N.; Sasaki, K.; Sastry, T.V.R.S.; Kanai, M.; Shibasaki, M. J. Org. Chem., 2006, 71, 1220. (h) Balskus, E.P.; Jacobsen, E.N. J. Am. Chem. Soc., 2006, 128, 6810 .

[6] Sano, S.; Liu, X.-K.; Takebayashi, M.; Kobayashi, Y.; Tabata, K.; Shiro, M.; Nagao, Y. Tetrahedron Lett., 1995, 36, 4101. (b) Sano, S.; Takebayashi, M.; Miwa, T.; Ishii, T.; Nagao, Y. Tetrahedron: Asymmetry, 1998, 9, 3611. (c) Sano, S.; Miwa, T.; Liu, X.-K.; Ishii, T.; Takehisa, T.; Shiro, M.; Nagao, Y. Tetrahedron: Asymmetry, 1998, 9, 3615. (d) Sano, S.; Ishii, T.; Miwa, T.; Nagao, Y. Tetrahedron Lett., 1999, 40, 3013.

[7] (a) Sano, S.; Hayashi, K.; Miwa, T.; Ishii, T.; Fujii, M.; Mima, H.; Nagao, Y. Tetrahedron Lett., 1998, 39, 5571. (b) García-Urdiales, E.; Alfonso, I.; Gotor, V. Chem. Rev., 2005, 105, 313.

[8] Sano, S.; Ushirogochi, H.; Morimoto, K.; Tamai, S.; Nagao, Y. Chem. Commun., 1996, 1775. (b) Zanoni, G.; Castronovo, F.; Franzini, M.; Vidari, G.; Giannini, E. Chem. Soc. Rev., 2003, 32, 115.

[9] Tsuji, T.; Iio, Y.; Takemoto, T.; Nishi, T. Tetrahedron: Asymmetry, 2005, 16, 3139. (b) Sano, S.; Nakao, M.; Takeyasu, M.; Honjo, T.; Nagao, Y. Lett. Org. Chem., 2006, 3, 764. (c) Tian, P.; Xu, M.-H.; Wang, Z.-Q.; Li, Z.-Y.; Lin, G.-Q. Synlett, 2006, 1201. (d) Miyaoka, H.; Yamanishi, M.; Hoshino, A.; Kinbara, A. Tetrahedron, 2006, 62, 4103. (e) Nakamura, T.; Tsuji, T.; Iio, Y.; Miyazaki, S.; Takemoto, T.; Nishi, T. Tetrahedron: Asymmetry, 2006, 17, 2781.

[10] Pihko, A.J.; Lundell, K.; Kanerva, L.: Koskinen, A.M.P. Tetrahedron: Asymmetry, 2004, 15, 1637. (b) Hazelard, D.; Fadel, A.; Morgant, G. Tetrahedron: Asymmetry, 2004, 15, 1711. (c) Levy, L.M.; de Gonzalo, G.; Gotor, V. Tetrahedron: Asymmetry, 2004, $15,2051$.

[11] Middleton, W.J. J. Org. Chem., 1975, 40, 574. (b) Kaduk, C.; Wenschuh, H.; Beyermann, M.; Forner, K.; Carpino, L.A.; Bienert, M. Lett. Peptide Sci., 1995, 2, 285. (c) Horikawa, M.; Nakajima, T.; Ohfune, Y. Synlett, 1997, 253. (d) Davis, F.A.; Zhang, Y.; Rao, A.; Zhang, Z. Tetrahedron, 2001, 57, 6345. (e) Lee, J.; Lee, Y.-I.; Kang, M.J.; Lee, Y.-J.; Jeong, B.-S.; Lee, J.-H.; Kim, M.-J.; Choi, J.-y.; Ku, J.-M.; Park, H.-g.; Jew, S.-s. J. Org. Chem., 2005, 70, 4158 .

[12] (a) Moon, S.-H.; Ohfune, Y. J. Am. Chem. Soc., 1994, 116, 7405. (b) Avenoza, A.; Cativiela, C.; Corzana, F.; Peregrina, J.M.; Sucunza, D.; Zurbano, M.M. Tetrahedron: Asymmetry, 2001, 12, 949.

[13] Sano, S.; Nakao, M.; Takeyasu, M.; Kitaike, S.; Yoshioka, Y.; Nagao, Y. Heterocycles, 2009, 79, in press.

[14] (a) Isobe, M.; Ichikawa, Y.; Goto, T. Tetrahedron Lett., 1986, 27, 963. (b) Isobe, M.; Ichikawa, Y.; Bai, D.-L.; Goto, T. Tetrahedron, 1987, 43, 4767.

[15] Zaidlewicz, M.; Brown, H.C. In Handbook of Reagents for Organic Synthesis-Oxidizing and Reducing Agents, Burke, S.D. and Danheizer, R.L., Ed.; Wiley: Chichester, 1999, pp. 227-230. 
[16] Shioiri, T.; Aoyama, T. In Handbook of Reagents for Organic Synthesis-Activating Agents and Protecting Groups, Pearson, A.J. and Roush, W.R., Ed.; Wiley: Chichester, 1999, pp. 422-425.

[17] (a) Fauq, A.H. In Handbook of Reagents for Organic SynthesisActivating Agents and Protecting Groups, Pearson, A.J. and Roush,
W.R., Ed.; Wiley: Chichester, 1999, pp. 137-140. (b) Kaduk, C.; Wenschuh, H.; Beyermann, M.; Forner, K.; Carpino, L.A.; Bienert, M. Lett. Peptide Sci., 1995, 2, 285.

Received: November 27, 2008

Revised: December 05, 2008

Accepted: December 05, 2008

(C) Sano et al.; Licensee Bentham Open.

This is an open access article licensed under the terms of the Creative Commons Attribution Non-Commercial License (http://creativecommons.org/licenses/by-nc/3.0/) which permits unrestricted, non-commercial use, distribution and reproduction in any medium, provided the work is properly cited. 